\title{
1 A local insulin reservoir ensures developmental progression in condition of nutrient 2 shortage in Drosophila
}

3 Suhrid Ghosh ${ }^{1,2^{*}}$, Weihua Leng ${ }^{1}$, Michaela Wilsch-Bräuninger ${ }^{1}$, Pierre Léopold ${ }^{3 *}$ and Suzanne Eaton ${ }^{1,2}$

$4 \quad{ }^{1}$ Max Planck Institute of Molecular Cell Biology and Genetics, Pfotenhauerstraße 108

501307 Dresden, Germany

6 2Biotechnologisches Zentrum, Technische Universität Dresden, Tatzberg 47/49, 01307 Dresden, Germany.

$7 \quad$ IInstitut Curie, PSL Research University, CNRS UMR3215, INSERM U934, 26 Rue d'Ulm, 75005 Paris, France.

8 *Authors for correspondence: ghosh@mpi-cbg.de, pierre.leopold@curie.fr

11 Summary (129 words, max 150)

12 Insulin/IGF signalling (IIS) controls many aspects of development and physiology. In Drosophila, a conserved

13 family of insulin-like peptides (Ilp) is produced by brain neurosecretory cells and exerts systemic functions.

14 Here, we describe the local uptake and storage of Ilps in the Corpora Cardiaca (CC), a group of alpha cell 15 homolog that produces the glucagon-like hormone AKH. Dilp uptake relies on the expression of Impl2, an

16 IGF-BP that accumulates in the CCs. During nutrient shortage, this specific reserve of Ilps is released and

17 activates IIS in a paracrine manner in the prothoracic gland, securing accelerated entry into pupal

18 development through the production of the steroid hormone ecdysone. We therefore uncover a sparing

19 mechanism whereby local Ilp storage and release activates the production of steroids and ensures early

20 developmental progression in adverse food conditions.

21

\section{Highlights}

23 - Dilps are uptaken by CC cells through the IGF-BP Imp-L2

24 - the CC-Dilp store is released upon nutrient shortage and activates IIS through CC projections on the PG

25 - upon nutrient shortage, IIS activation in the PG ensures an accelerated transition from larval feeding stage

26 to metamorphosis.

\section{Keywords}

29 insulin, ecdysone, nutrition, hormone secretion, Drosophila 


\section{Introduction}

32 Insulin and insulin-like growth factors (IGFs) are conserved modulators of growth and metabolic

33 homeostasis. They are produced by specific endocrine organs in response to nutrient availability and

34 stimulate peripheral tissues through two main routes. Circulating insulins and IGFs act in an endocrine

35 manner on distant organs through mechanisms of secretion and action that have been extensively studied

36 (Campbell \& Newgard, 2021; Petersen \& Shulman, 2018; Tokarz, MacDonald, \& Klip, 2018). In several

37 instances, insulin/IGFs are also produced and act locally. This is the case for IGF-I produced in peripheral

38 organs like bone chondrocytes, which acts in an autocrine manner (Wang, Bikle, \& Chang, 2013). Local

39 insulin/IGF signalling is also key for the development and homeostasis of the central nervous system, which

40 is separated from the systemic circulation by the blood-brain barrier (Fernandez \& Torres-Alemán, 2012).

41 In mammalian brains, IGF-I expression is spatially and temporally controlled, participating in the rapid

42 proliferation of neural stem cells (NSCs) (Popken et al., 2004). IGF-I is expressed in neurons, astrocytes and

43 NSCs, contributing to both paracrine and autocrine signaling ( $D^{\prime}$ Ercole \& Ye, 2008).

44 In the Drosophila model, a subset of insulin/IGFs called Drosophila insulin-like peptides (Dilps) is expressed

45 in neurosecretory cells and contributes to systemic activation of insulin/IGF signaling (IIS). Similar to

46 mammals, local brain expression of Dilps in glial cells also contributes to coupling NSC proliferation with

47 systemic metabolic status (Yuan, Sipe, Suzawa, Bland, Siegrist \& Id, 2020; Spéder \& Brand, 2018).

48 In parallel, local activation of IIS can be triggered by alternative routes, providing the possibility to uncouple

49 insulin/IGF signaling from nutrient availability. The role of this uncoupling is exemplified in the case of "brain

50 sparing" a phenomenon observed in many species, including human, whereby moderate nutrient

51 deprivation affects primarily body growth, while brain growth is preserved (Cox \& Marton, 2009). The

52 molecular mechanism of brain sparing has been addressed in Drosophila. It is associated with the specific

53 activation of neuroblast growth through local production of the ligand Jelly belly (Jeb) and activation of the

54 Anaplastic lymphoma kinase (Alk) receptor upstream of PI-3-kinase, even in the absence of nutrients (Cheng

55 et al., 2011).

56 Here, we unravel a distinct mechanism locally uncoupling insulin release from the presence of nutrients and

57 leading to the maintenance of IIS activation in a key endocrine organ during development. The initial

58 observation of a pool of Dilps accumulating in an endocrine gland called the corpora cardiaca (CC) (S. K. Kim

$59 \&$ Rulifson, 2004), suggested the possibility that these insulins could activate IIS under specific conditions,

60 in a way distinct from their systemic function. Indeed, the CC cells do not produce insulins, but rather a

61 glucagon-like hormone called $\mathrm{AKH}$, whose release is stimulated by nutrient shortage. Our present work

62 unravels some features of the mechanism of uptake of insulins by the CCs. We show that, conversely to

63 systemic insulins, CC insulins are released in response to nutrient deprivation. We further demonstrate that 
64 the local release of CC insulins upon starvation maintains high IIS levels in the prothoracic gland (PG), which

65 produces the steroid ecdysone. Finally, we establish that the local activation of IIS in the PG secures a fast

66 transition to pupal development in absence of nutrients.

67 Therefore, our work uncovers a distinct mechanism of organ-sparing, whereby a pre-accumulated store of

68 Dilps is released in adverse food conditions and ensures fast developmental progression through steroid

69 production.

\section{Results}

\section{Corpora cardiaca cells take up Dilp2 and 5}

The Corpora Cardiaca (CC) is part of the ring gland (RG), a composite endocrine organ located in the vicinity of the larval central nervous system (CNS) (Figure 1a). Apart from the CC, two other endocrine

75 tissues - the prothoracic gland (PG), which synthesizes ecdysone, and the corpora allata (CA), which 76 synthesizes the juvenile hormone - together form the PG (Figure 1b). CC cells are neurosecretory in nature and extend processes that terminate in the larval aorta and the PG (Figure 1b). The IPCs, located in the pars intercerebralis of the larval brain, also project to the aorta (Figure 1b). Previous work describes a strong accumulation of anti-Dilp2 immunoreactivity in the corpora cardiaca (CC) (S. K. Kim \& Rulifson, 2004), in

80 addition to the one observed in the cell bodies of IPCs (Rulifson, 2002). However, whether CC cells express 81 the Dilp2 gene has not been investigated.

In order to clarify the presence and production of Dilp2 in CC cells, we co-labelled the IPC 83 projections by over-expressing a GFP-tagged Synaptotagmin-1 (SYT1-GFP) using a dilp2-gal4 driver

84 (IPC>SYT1-GFP, Figure 1c) and anti-Dilp2 immunostaining. We confirmed the presence of IPC processes 85 containing DILP2 and innervating the aorta (Figure 1c). Additionally, Dilp2 could also be detected without 86 GFP colocalization in the CC soma (Figure 1c). Expressing SYT1-GFP using a CC-specific GAL4 driver (akh87 ga/4), we confirmed that Dilp2 indeed co-localizes with GFP in CC cells and their processes projecting on 88 the PG and the aorta (Figure 1d). CC soma also stain for anti- Dilp5, and both Dilp2 and Dilp5 show high 89 degree of subcellular colocalization (Figure 1e), suggesting that these two Dilps may serve a common 90 function in the CCs. Their presence in the soma of the CC cells could either be due to a transient expression

91 in the CCs or their uptake by the CCs from a pool initially produced by the IPCs. To address this point, we 92 specifically knock-down DILP2 or DILP5 expression in the IPCs. This led to their loss in CC cells (Figures 1f-i), 93 indicating that the IPCs are the only source of Dilp production, followed by their specific uptake in the CCs.

94 Of note, the knock-down of Dilp5 expression in the IPCs do not affect the uptake of Dilp2 by the CCs (Figure $95 \mathrm{~S} 1)$. 


\section{IMPL2 mediates the uptake of Dilp2 and Dilp5 in the Corpora cardiaca}

98 In addition to the canonical insulin receptor (dlnR), Drosophila larvae express another insulin-binding

99 protein called IMPL2. IMPL2 binds circulating Dilp2 and 5, resulting in the downregulation of insulin/IGF-

100 signalling (IIS) in target tissues (Figueroa-Clarevega \& Bilder, 2015). Beside tumorous imaginal discs and fat

101 body (Figueroa-Clarevega \& Bilder, 2015; Lee et al., 2018), which produce a circulating form of ImpL2, a

102 subset of neurons in the larval brain express the IMPL2 gene (Honegger et al., 2008). Using specific anti-

$103 \mathrm{ImpL2}$ antibodies, we also detected IMPL2 in the CCs (Figure 2a). Similar staining patterns between IMPL2

104 and Dilp2 suggests that they may be partially bound inside the CC. Staining for IMPL2 in Dilp2/Dilp5 double

105 knock-out larvae ruled out a possible staining artefact due to cross-reactivity of the respective antibodies

106 (Figure S2). Additionally, our results show that the presence of IMPL2 in the CC is independent of Dilp2 and

1075 production (Figure S2).

108 IMPL2 binds to Dilps but, unlike dlnR, does not have a trans-membrane domain (Roed et al., 2018). A 109 previous study (Bader et al., 2013) showed that a membrane-associated form of IMPL2 produced in a few

110 brain neurons binds Dilp2 and facilitates its contact with dlnR, resulting in activation of intracellular IIS 111 signalling. While dInR is recycled to the membrane after endocytosis, the IMPL2-Dilp2 complex is then 112 degraded (Bader et al., 2013). In order to clarify the mechanism of Dilp uptake by the CCs, we knocked113 down dInR and IMPL2 and compared the amount of Dilps present in CC cells. dInR and IMPL2 knock-down 114 were first controlled for their efficacies in the wing disc and CC respectively (Figure S4 and S5a). While 115 silencing $d I n R$ in CCs had no effect on Dilp2 localization, ImpL2 knock-down significant reduced Dilp2 116 staining in the CC soma (Figures 2b and 2c). Similar results were obtained for Dilp5 localisation (Figure S3).

117 The knock-down of $d I n R$ reduces CC cell area and shows a slight increase in mean intensities of Dilp2 (Figures 118 2c) and 5 (Figures S3). Silencing Impl2 in CCs did not affect expression of Impl2 in brain neurons (Figures $119 \mathrm{~S} 5 \mathrm{~b})$. We conclude from these experiments that CC cells require IMPL2, but not dInR, to take up Dilps. We 120 next ensured that preventing Dilp uptake in the CC does not affect endogenous glucagon-like AKH production by staining CCs with anti-AKH antibodies (Figures $2 \mathrm{~d}$ and 2e). Therefore, Impl2 silencing in CC cells is an efficient tool to study the physiological function of the pool of Dilps present in the CCs.

124 Corpora cardiaca act as a functional Dilp store

125 Larvae lacking Dilp2 and Dilp5 show reduced growth during larval stages, require extended time to develop 126 and produce smaller adults (Grönke, Clarke, Broughton, Andrews, \& Partridge, 2010). To evaluate the 127 contribution of the CC store of Dilp2 and Dilp5 to general IIS, we measured the developmental time, larval 128 growth and adult size of animals devoid of Impl2 in CC cells, therefore lacking Dilp accumulation in CCs. We 129 observed that larvae of the CC>Imp/2-RNAi genotype show approx. $10 \mathrm{hr}$ advanced pupariation compared 
130 to controls (Figure 3a). However, pupal volume, as a measure of total larval growth, adult mass and wing

131 area are not modified (Figure 3b-d). This indicates that animals devoid of a CC-Dilp store experience

132 accelerated growth without changing final size. Measuring larval mass across the L3 stage, we indeed found

133 that removal of CC-Dilps leads to an increase in larval growth rate (Figure 3e). Accelerated growth was

134 previously observed upon general increase in intracellular IIS (Brogiolo et al., 2001) or over-expression of

135 DILP2 (Géminard, Rulifson, \& Léopold, 2009; Ikeya et al., 2002) during larval development. When measuring

136 circulating Dilp2 from CC>Imp/2-RNAi larvae, we found it doubling compared to controls (Figure 3f). To

137 support this finding, we also found elevated levels of fat body IIS at 104hr AEL compared to control

138 genotypes, measured as the relative distribution of the transcription factor dFOXO between nucleus and

139 cytoplasm (Figure 3g and 3h). Therefore, preventing DILP uptake in the CC increases circulating Dilp2 levels,

140 resulting in accelerated growth.

141

142 CC-Dilps are packaged for co-secretion with AKH

143 Our previous results indicate that approximately equal amounts of Dilp circulate in the hemolymph and are

144 sequestered in the CC cells (Figure 3f). We therefore studied the control of secretion of this major pool of

145 CC-Dilp. Based on colocalization using light microscopy, it was previously proposed that in ImpL2-expressing

146 neurons, ImpL2/Dilp2 complexes are degraded through the late endosomal route (Bader et al., 2013). Given

147 the small size of the CC soma, we used electron microscopy to identify DILP-containing structures inside the

148 CC cells (Figure 4a). Stitching several high-resolution images from a single tissue section shows the overall

149 organization of the CC cytoplasm (Figure 4b). The cytoplasm contains large networks of ER, occasionally

150 interspersed with Golgi complexes, and clusters of dense-core secretory vesicles (DCVs) occupying the

151 perinuclear space (Figure 4b). This architecture is in line with the capacity of CC cells to secrete the

152 glucagon-like hormone AKH, and similar observations have been made in other insects (Willey \& Chapman,

153 1960). When probed for Dilp5 using immuno-gold staining, CC cells showed high reactivity in clusters of

154 DCVs compared to rest of the cytoplasm (Figures 4c and 4d). Probing simultaneously for Dilp2 and 5 using

155 gold particles of two different sizes, we observed a co-localization of both Dilps in DCVs, but also in multi-

156 vesicular bodies (MVBs) (Figure 4e). MVBs form in the late endocytic pathway and are responsible for

157 recycling and degradation of endocytic compartments (Cosker \& Segal, 2014; Sorkin \& Von Zastrow, 2009).

158 Alternatively, they also give rise to secretory granules (Gondré-Lewis, Park, \& Loh, 2012). Our results

159 therefore suggest that Dilp2 and 5 are taken up and packaged into secretory DCVs, making the CC cells a

160 potential alternative source of insulin in the Drosophila larva.

161 CC cells endogenously produce the glucagon-like peptide hormone AKH and release it under nutrient-

162 limiting conditions (S. K. Kim \& Rulifson, 2004). When probing CC cells for AKH and Dilp2 using two 
163 differently-sized gold particles, we found that AKH and Dilp2 co-localize in the same DCVs (Figure 4f). We

164 also noted that AKH seems more abundant than Dilp2 in these DCVs (Figure 4g). Therefore, two functionally

165 antagonistic hormones, AKH and Insulins, are co-packaged in the same secretory structures, raising the

166 intriguing possibility that they are released by CC cells in the same physiological context (i.e. limiting

167 nutrients).

168

169 CC-Dilps control the time to pupariation under nutrient limitation.

170 What could be the physiological role of the pool of CC-insulin? During the late larval period, animals pass

171 through a nutrient restriction checkpoint (NRC), beyond which larvae are committed to pupal development

172 even when fully starved (Pan, Neufeld, Connor, et al., 2019). Early observations (Beadle et. al., 1938)

173 indicate that when starved after the NRC, Drosophila larvae pupariate earlier than fed controls, as we

174 observed for $C C>w 1118$ (Figure 5a,b).

175 Several lines of evidence suggest that the pool of CC-Dilps induces early pupariation upon starvation post-

176 NRC. First, we noticed that, when comparing fed and starved conditions, the acceleration observed for

$177 C C>W 1118$ controls is absent in the CC>ImpL2-RNAi background (Figure 5c). As described before (see Figure

$1783 a, e)$, fed $C C>$ ImpL2-RNAi larvae pupariate earlier than fed CC> controls due to accelerated growth, but in

$179 C C>I m p L 2-R N A i$ larvae, the difference between fed and starved conditions is abrogated (marked as a grey

180 area in Figures $5 b, c$ ). We then confirmed that CC-Dilps are released upon low/no nutrient conditions (Figure

$1815 \mathrm{~d}-\mathrm{g}$ ), reminiscent of what is observed for the control of AKH secretion (J. Kim \& Neufeld, 2015). The

182 transition into pupal development requires a surge of ecdysone production and previous work indicate that

183 activation of IIS is required for ecdysone biosynthesis by the PG (Boulan, Martín, \& Milán, 2013; Colombani,

184 2005). Therefore, our observation that CC cells send projections to the PG raises the possibility of a local

185 delivery of Dilps to the PG in response to starvation. To test this idea, we compared the levels of circulating

186 Dilp2 in CC> and CC>ImpL2-RNAi larvae under different nutritional conditions. In fed conditions, suppressing

187 the CC-Dilp pool leads to an increase in circulating Dilp2 (Figure 5h). However, during starvation post-NRC,

188 no circulating Dilp2 is detected either in CC> or CC>Imp/2 conditions (Figure 5i), indicating that the release

189 of the CC pool of Dilp2 does not contribute to increasing systemic Dilp2. In parallel, we measured the

190 nucleus/cytoplasm ratio of dFOXO in fat cells as a marker of peripheral IIS (Figure 5j,k). Again, no difference

191 in adipose IIS was observed between $C C>$ and $C C>I m p L 2-R N A i$ larvae starved after the NRC, indicating that

192 under these conditions, CC-Dilp2 does not contribute to general IIS. In contrast, when conducting a similar

193 analysis of the cells of the prothoracic gland, which synthesizes ecdysone, we observe that starvation after

194 the NRC induces an increase in nuclear localization of dFOXO in the PG of CC>ImpL2-RNAi but not of CC>

195 control animals (Figure 5l, m). This result indicates that CC-Dilps contribute to maintaining a high level of IIS 
196 activation in PG cells in response to post-CW starvation. In line with our finding that CC>ImpL2-RNAi larvae

197 pupariate earlier under these conditions, we conclude that the pool of CC-Dilps contributes to a local

198 activation of IIS in PG cells, maintaining their capacity to synthesize ecdysone and allowing a premature 199 transition into pupal development in response to late starvation.

\section{Discussion}

203 In this study, we re-evaluate a previous observation whereby glucagon-producing cells take up insulin

204 peptides, and demonstrate the relevance of this phenomenon during Drosophila larval development. In the 205 course of early studies on Drosophila insulin-producing cells (S. K. Kim \& Rulifson, 2004), it was indeed noted 206 that larval CC cells strongly label for Dilp2 peptides. This puzzling observation suggested that the larval CC 207 could serve the role of a so-called "neurohemal" organ, storing Dilps for delivery in the general circulation 208 under specific conditions. However, the mechanistic and functional aspects of this Dilp store were unknown. 209 Our study now sheds new light on the role of CC-Dilps, linking them to another unexplained observation, 210 the starvation-induced, premature transition of Drosophila larvae into pupal development (Beadle et. al., 211 1938).

\section{Accelerating development upon post-critical weight starvation}

213 Drosophila populations can suffer episodes of nutrient limitation, particularly during the larval period when 214 mobility of individuals is limited, preventing them from exploring alternative food sources. When larvae 215 endure full starvation passed the so-called nutrient restriction checkpoint (NRC), an acceleration of 216 pupariation is observed and animals transit faster to an adult reproductive state, with a trade-off on 217 individual size. This contrasts with the observation that Dilp secretion is inhibited by starvation (Géminard 218 et al., 2009), leading to a reduction of circulating hormone levels. Such reduction should indeed affect the 219 production of ecdysone, which relies on IIS in PG cells (Colombani, 2005). Our present work indicates that 220 upon limiting nutrients, CC cells secrete stored Dilps to the PG in a paracrine fashion (through PG-specific projections) without affecting systemic IIS. The CC-derived Dilps sustain IIS in PG cells, providing the 222 necessary signalling input for ecdysone production and accelerated pupariation. Indeed, IIS activation could 223 prevent starvation-induced autophagy in PG cells, which has been shown to block ecdysone production in 224 condition of a pre-NRC starvation, by shunting cholesterol away from the biosynthetic pathway (Pan, 225 Neufeld, \& O'Connor, 2019). Recent evidence suggests that alternative nutrient-independent growth 226 factors such as Jelly-belly (Jeb) can also act on PG cells to stimulate IIS and ecdysone production (Pan \& 
removal of CC Dilps only affects acceleration upon nutrient restriction but does not delay pupariation. Whether CC-Dilps are uniquely targeted to the PG, and through which mechanism, are still open questions.

231 Storing Dilps away from general circulation

232 We find that preventing CC-Dilp storage increases circulating levels by 2 -fold. This accelerates animal's 233 growth rate during the late larval stages, when circulating Dilp levels wane (compare Figures $3 f$ and $5 \mathrm{~h}$; also 234 see Okamoto \& Nishimura, 2015). We also noted that such accelerated larval growth does not affect adult 235 size due a compensatory advance in pupariation. This result suggests an interdependency relationship 236 between the two Dilp reservoirs, whereby the CC-Dilp store forms from ILP-produced insulins that are 237 derouted from the general circulation in conditions of optimal nutrition. It has been described previously 238 that the IPC project their termini in proximity of the CCs onto the aorta. Whether CC-Dilp accumulates 239 through local delivery of IPC-produced Dilps or by uptake from the general circulation is still unknown.

240 Insulins bind to their cognate receptor InR in target tissues and are then co-endocytosed and later degraded 241 (Sopko \& Perrimon, 2013; Sorkin \& Von Zastrow, 2009). Our study demonstrates that the uptake of Dilps by 242 CC cells does not rely on InR but rather Imp-L2, an alternative insulin-binding protein previously shown to 243 bind circulating Dilps.-This result contrasts with the previously described case of Imp-L2-producing Hugin 244 neurons in the larval CNS, where both InR and Imp-L2 are required for Dilp uptake (Bader et al., 2013). This 245 difference might have important functional consequences. In contrast to endocytosed Dilps/Inr complexes, 246 which are prone to degradation, endocytosed ImpL2/Dilp complexes are routed towards storage in DCV, in 247 the absence of local activation of IIS. Dilps are co-packaged with AKH in DCVs, possibly during the DCV 248 maturation phase. This phase is characterized by cargo exchange between endo-lysosomal compartments 249 and immature vesicles budding off from the trans-Golgi network (Topalidou et al., 2016). Recent studies 250 (Gondré-Lewis et al., 2012; Lund, Lycas, Schack, Andersen, \& Gether, 2020) suggest that RAB2 and 251 associated proteins are involved in this exchange. However, we do not know if these components 252 participate in routing DILPs and AKH to DCVs in the CC. Further, Dilps are anterogradely transported along 253 the axonal projections in the PG. This process is similar to the transcytosis of various trophic factors and 254 pathogens observed in mammalian nervous systems (Von Bartheld, 2004). Unlike simultaneous uptake and 255 release previously demonstrated in other neuron types (Hémar, Olivo, Williamson, Saffrich, \& Dotti, 1997; 256 Von Bartheld, Wang, \& Butowt, 2001; Yamashita, Joshi, Zhang, Zhang, \& Kuruvilla, 2017), we show here that 257 Dilp uptake and release are temporally separated during larval development. In all, this sheds light on the 258 unique ability of $\mathrm{CC}$ neurosecretory cells to convert an endocrine signal like insulin into a paracrine one. 
260 In conclusion, we reveal here a mechanism of organ sparing relying on the storage and release of insulins,

261 allowing the maintenance of IIS activation and ecdysone production in a paracrine organ. The existence of

262 such mechanism opens the intriguing possibility that local pools of insulin/IGF could be used in response to

263 environmental signals to maintain IIS signalling in spared organs in other models.

264

265

\section{Acknowledgements}

267 This article is dedicated to the memory of our great colleague and mentor Prof./Dr. Suzanne Eaton. We are 268 grateful to Dr. Natalie Dye for her supervision and leadership. We thank Dr.Jonathan Rodenfels, the Eaton 269 and Leopold lab members for insightful discussions and comments on the manuscript. We would also like 270 to thank the BDSC, Prof. Linda Partridge and Dr. Jason Tennessen for providing us with fly lines, Prof. Marc 271 Tatar and Dr. Stephanie Post for sharing reagents, and Dr. Hugo Stocker for gifting us the Imp-L2 antibody. 272 The scientific illustrations were done by Dr. Bertsy Goic. This work was supported by Deutsche 273 Forschungsgemeinschaft (DFG/FOR2682), Max-Planck-Gesellschaft and Institut Curie, INSERM.

\section{Author contributions}

276 Conceptualization, S.G., S.E., P.L., Methodology, S.G., S.E., P.L., Investigation, S.G., W.L., M.W.-B., Writing 277 and editing, S.G., P.L., Funding Acquisition, S.E.; Supervision, S.E., P.L.

\section{Declaration of interests}

280 The authors declare no competing interests.

\section{References}

284 Bader, R., Sarraf-Zadeh, L., Peters, M., Moderau, N., Stocker, H., Kö hler, K., ... Hafen, E. (2013). The IGFBP7 285 homolog Imp-L2 promotes insulin signaling in distinct neurons of the Drosophila brain. Journal of Cell Science, 126(12), 2571-2576. https://doi.org/10.1242/jcs.120261

Boulan, L., Martín, D., \& Milán, M. (2013). Bantam miRNA promotes systemic growth by connecting insulin signaling and ecdysone production. Current Biology, 23(6), 473-478. https://doi.org/10.1016/j.cub.2013.01.072

290 Brogiolo, W., Stocker, H., Ikeya, T., Rintelen, F., Fernandez, R., \& Hafen, E. (2001). An evolutionarily conserved function of the drosophila insulin receptor and insulin-like peptides in growth control. 
Campbell, J. E., \& Newgard, C. B. (2021). Mechanisms controlling pancreatic islet cell function in insulin secretion. Nature Reviews Molecular Cell Biology, 22(2), 142-158. https://doi.org/10.1038/s41580020-00317-7

Cardona, A., Saalfeld, S., Schindelin, J., Arganda-Carreras, I., Preibisch, S., Longair, M., ... Douglas, R. J. (2012). TrakEM2 software for neural circuit reconstruction. PLOS ONE, 7(6). https://doi.org/10.1371/journal.pone.0038011

299 Cheng, L. Y., Bailey, A. P., Leevers, S. J., Ragan, T. J., Driscoll, P. C., \& Gould, A. P. (2011). Anaplastic

Colombani, J. (2005). Antagonistic Actions of Ecdysone and Insulins Determine Final Size in Drosophila. Science, 310(5748), 667-670. https://doi.org/10.1126/science.1119432

Cosker, K. E., \& Segal, R. A. (2014). Neuronal signaling through endocytosis. Cold Spring Harbor Perspectives in Biology, 6(2). https://doi.org/10.1101/cshperspect.a020669

Cox, P., \& Marton, T. (2009). Pathological assessment of intrauterine growth restriction. Best Practice and https://doi.org/10.1016/j.bpobgyn.2009.06.006

D’Ercole, A. J., \& Ye, P. (2008). Minireview: Expanding the mind: Insulin-like growth factor I and brain development. Endocrinology, 149(12), 5958-5962. https://doi.org/10.1210/en.2008-0920

Fernandez, A. M., \& Torres-Alemán, I. (2012). The many faces of insulin-like peptide signalling in the brain.

Figueroa-Clarevega, A., \& Bilder, D. (2015). Malignant drosophila tumors interrupt insulin signaling to induce https://doi.org/10.1016/j.devcel.2015.03.001

Géminard, C., Rulifson, E. J., \& Léopold, P. (2009). Remote Control of Insulin Secretion by Fat Cells in Drosophila. Cell Metabolism, 10(3), 199-207. https://doi.org/10.1016/j.cmet.2009.08.002 Functional Characterization of Drosophila Insulin-Like Peptides. PLoS Genetics, 6(2), 18. https://doi.org/10.1371/journal.pgen.1000857

Hémar, A., Olivo, J. C., Williamson, E., Saffrich, R., \& Dotti, C. G. (1997). Dendroaxonal transcytosis of 
transferrin in cultured hippocampal and sympathetic neurons. Journal of Neuroscience, 17(23), 90269034. https://doi.org/10.1523/jneurosci.17-23-09026.1997

Honegger, B., Galic, M., Köhler, K., Wittwer, F., Brogiolo, W., Hafen, E., \& Stocker, H. (2008). Imp-L2, a putative homolog of vertebrate IGF-binding protein 7, counteracts insulin signaling in Drosophila and is essential for starvation resistance. Journal of Biology, 7(3). https://doi.org/10.1186/jbiol72

Id, X. Y., Id, C. W. S., Suzawa, M., Id, M. L. B., \& Id, E. S. (2020). Dilp-2 - mediated PI3-kinase activation coordinates reactivation of quiescent neuroblasts with growth of their glial stem cell niche, 1-23. https://doi.org/10.1371/journal.pbio.3000721

Ikeya, T., Galic, M., Belawat, P., Nairz, K., Hafen, E., \& Zu, C.-. (2002). Nutrient-Dependent Expression of Insulin-like Peptides from Neuroendocrine Cells in the CNS Contributes to Growth Regulation in Drosophila, 12(02), 1293-1300.

Isabel, G., Martin, J. R., Chidami, S., Veenstra, J. A., \& Rosay, P. (2005). AKH-producing neuroendocrine cell

Kakanj, P., Moussian, B., Grönke, S., Bustos, V., Eming, S. A., Partridge, L., \& Leptin, M. (2016). Insulin and ablation decreases trehalose and induces behavioral changes in Drosophila. American Journal of Physiology - Regulatory Integrative and Comparative Physiology, 288(2 57-2), 531-538. https://doi.org/10.1152/ajpregu.00158.2004

Kim, S. K., \& Rulifson, E. J. (2004). Conserved mechanisms of glucose sensing and regulation by Drosophila corpora cardiaca cells, 431(September), 316-320. https://doi.org/10.1038/nature02913.1.

Lee, G. J., Han, G., Yun, H. M., Lim, J. J., Noh, S., Lee, J., \& Hyun, S. (2018). Steroid signaling mediates nutritional regulation of juvenile body growth via IGF-binding protein in Drosophila. Proceedings of the National Academy of Sciences of the United States of America, 115(23), 5992-5997. dense core vesicles and lysosomal organelles, 1-52. Dependent Production of an Insulin-like Peptide for Drosophila Body Growth. Developmental Cell, 35(3), 295-310. https://doi.org/10.1016/j.devcel.2015.10.003 
Drosophila Pre-metamorphic Nutritional

Checkpoints.

Current

Biology,

$1-12$. https://doi.org/10.1016/j.cub.2019.07.027

Pan, X., Neufeld, T. P., \& O'Connor, M. B.

(2019). Controls Drosophila Pre-metamorphic Nutritional Checkpoints. Current Biology, 29(17), 2840-2851.e4. https://doi.org/10.1016/j.cub.2019.07.027

Pan, X., \& O'Connor, M. B. (2020). Coordination among multiple receptor tyrosine kinase signals controls drosophila developmental timing https://doi.org/10.1101/2020.09.01.278382

Park, S., Alfa, R. W., Topper, S. M., Kim, G. E. S., Kockel, L., \& Kim, S. K. (2014). A Genetic Strategy to Measure

Petersen, M. C., \& Shulman, G. I. (2018). Mechanisms of insulin action and insulin resistance. Physiological Circulating Drosophila Insulin Reveals Genes Regulating Insulin Production and Secretion. PLoS Genetics, 10(8). https://doi.org/10.1371/journal.pgen.1004555

Roed, N. K., Viola, C. M., Kristensen, O., Schluckebier, G., Norrman, M., Sajid, W., ... Brzozowski, A. M. (2018). Structures of insect Imp-L2 suggest an alternative strategy for regulating the bioavailability of insulinlike hormones. Nature Communications, 9(1). https://doi.org/10.1038/s41467-018-06192-3

Rulifson, E. J. (2002). Ablation of Insulin-Producing Neurons in Flies: Growth and Diabetic Phenotypes. Science, 296(5570), 1118-1120. https://doi.org/10.1126/science.1070058

Schindelin, J., Arganda-Carreras, I., Frise, E., Kaynig, V., Longair, M., Pietzsch, T., ... Cardona, A. (2012). Fiji: An open-source platform for biological-image analysis. Nature Methods, 9(7), 676-682. https://doi.org/10.1038/nmeth.2019

Sopko, R., \& Perrimon, N. (2013). Receptor tyrosine kinases in Drosophila development. Cold Spring Harbor Perspectives in Biology, 5(6), 1-31. https://doi.org/10.1101/cshperspect.a009050

Sorkin, A., \& Von Zastrow, M. (2009). Endocytosis and signalling: Intertwining molecular networks. Nature Reviews Molecular Cell Biology, 10(9), 609-622. https://doi.org/10.1038/nrm2748

Spéder, P., \& Brand, A. H. (2018). Systemic and local cues drive neural stem cell niche remodelling during neurogenesis in drosophila. ELife, 7, 1-16. https://doi.org/10.7554/eLife.30413

Tennessen, J. M., Baker, K. D., Lam, G., Evans, J., \& Thummel, C. S. (2011). The Drosophila estrogen-related receptor directs a metabolic switch that supports developmental growth. Cell Metabolism. 
Tokarz, V. L., MacDonald, P. E., \& Klip, A. (2018). The cell biology of systemic insulin function. Journal of Cell Biology, 217(7), 2273-2289. https://doi.org/10.1083/jcb.201802095

394 Topalidou, I., Cattin-Ortolá, J., Pappas, A. L., Cooper, K., Merrihew, G. E., MacCoss, M. J., \& Ailion, M. (2016).

395 The EARP Complex and Its Interactor EIPR-1 Are Required for Cargo Sorting to Dense-Core Vesicles. PLoS Genetics, 12(5), 1-29. https://doi.org/10.1371/journal.pgen.1006074

Von Bartheld, C. S. (2004). Axonal Transport and Neuronal Transcytosis of Trophic Factors, Tracers, and Pathogens. Journal of Neurobiology, 58(2), 295-314. https://doi.org/10.1002/neu.10315

Von Bartheld, C. S., Wang, X. X., \& Butowt, R. (2001). Anterograde axonal transport, transcytosis, and recycling of neurotrophic factors: The concept of trophic currencies in neural networks. Molecular Neurobiology, 24(1-3), 1-28. https://doi.org/10.1385/MN:24:1-3:001

Wang, Y., Bikle, D. D., \& Chang, W. (2013). Autocrine and Paracrine Actions of IGF-I Signaling in Skeletal Development. Bone Research, 1, 249-259. https://doi.org/10.4248/BR201303003

Willey, R. B., \& Chapman, G. B. (1960). The ultrastructure of certain components of the corpora cardiaca in orthopteroid insects. Journal of Ultrasructure Research, 4(1), 1-14. https://doi.org/10.1016/S00225320(60)80002-0

Yamashita, N., Joshi, R., Zhang, S., Zhang, Z. Y., \& Kuruvilla, R. (2017). Phospho-Regulation of Soma-to-Axon Transcytosis of Neurotrophin Receptors. Developmental Cell, 42(6), 626-639.e5. https://doi.org/10.1016/j.devcel.2017.08.009 


\section{Figure legends}

427 Figure 1. IPC-derived DILPs 2 and 5 accumulate in the corpora cardiaca.

428 (a-b) Neuroanatomy of the larval central nervous system and ring gland complex showing (b)innervation of 429 insulin-producing cells(IPC) and the corpora cardiaca(CC).

430 (c-d) Maximum z-volume projection of brain and ring gland complex of larvae expressing Synaptotagmin-1431 GFP (c.i and d.i, green) specifically in the (c) IPC (dilp2-gal4) or the (d) CC (akh-gal4). Tissues are co-stained 432 for DILP2 (c.ii and d.ii, magenta). Solid arrows denote aortal projections from (c) IPC (white arrow) and (d) 433 CC (yellow arrow). Open arrows (yellow) show CC projections to the PG. Merged channels (c.iii and d.iii) 434 channel show colocalization. Scale bar $=50 u m$

435 (e) Wild-type CC soma stained for neuronal marker HRP (e.i ; gray), DILP2 (e.ii, green) and DILP5 (e.iii, 436 magenta). Merged channel (e.iv) show DILP2 and DILP5 colocalization. $\mathrm{R}=$ pixel-based Pearson's correlation 437 coefficient between the same. Scale bar $=10$ um.

438 (f,h) CC soma of IPC-specific(dilp2-gal4) (f)DILP2 RNAi in Dicer2-expressing background or (h)DILP5 RNAi 439 (h.iv-vi) together with wild-type controls(f.i-iii and h.i-iii). Stained for neuronal marker HRP(f.i, f.iv, h.i and 440 h.iv, gray) and (f)DILP2 (f.ii and f.v, green) or (h)DILP5 (h.ii and h.v, green). Merged channels (f.iii, f.vi, h.iii 441 and h.iv) for clarity. Scale bar =10um.

442 (g, i) Average fluorescence intensity of (g)DILP2 or (i)DILP5 normalized to the control mean. Each point 443 represents a single CC soma. $n=3-7 ; * *=p<0.01$, Student's t-test.

445 Figure 2. IMPL2 in the corpora cardiaca is necessary for DILP uptake.

446 (a) CC soma specifically expressing(akh-gal4) Synaptotagmin-1-GFP (a.i, yellow); stained for nuclei (a.i,blue), 447 IMPL2 (a.ii, red) and DILP2 (a.iii, green).

448 (b) CC-specific(akh-gal4) RNAi-mediated knockdown of insulin receptor (b.iv-vi), IMPL2 (b.vii-ix) and wild449 type control (b.i-iii). Stained for neuronal marker HRP (b.i, b.iv, and b.vii, gray) and DILP2 (b.ii, b.v and b.viii, 450 green). Merged channels (b.iii, b.iv and b.ix) for clarity. 
451 (d) CC-specific(akh-gal4) RNAi-mediated knockdown of IMPL2 (d.vii-ix) and wild-type control (b.i-iii). Stained 452 for neuronal marker HRP (b.i, b.iv, and b.vii, gray) and AKH (d.ii,and d.iv, blue).

453 (c, e) Average fluorescence intensity of (c)DILP2 or (e)AKH normalized to the control mean. Each point 454 represents a single CC soma. $n=3-5 ; * *=p<0.01$, $n s=p>0.01$; Student's t-test.

455 Scale bar $=10 u m$.

\section{Figure 3. Corpora cardiaca sequesters circulating DILPs.}

459 (a) Percentage pupariated of indicated genotype at various timepoints. AEL= after egg-laying. $n=6$ for each 460 genotype.

461 (b) Pupal volume ( $n=24)$, (c) adult weight $(n=7,9)$ and (d) adult wing area $(n=55,20)$ normalized to control. 462 Each point represents a larva/fly in the case of pupal volume or wing area, and mean of a cohort of 8-15 463 flies in the case weight measurements.

464 (e) Larval mass of indicated genotype at specified developmental time $(n=8-12)$. Each point represents mean 465 of cohorts of 13-15 flies.

466 (f) DILP2-HA-FLAG concentration in larval hemolymph of indicated genotype using driver line akh-gal4, dilp2$467 \quad H F(n=7,5)$.

468 (g) Fat body of indicated genotype stained for nuclei (g.i and g.iii, blue) and mCherry (g.ii and g.iv, red). 469 White dashed outlines show the position of some nuclei in the mCherry channel. Scale bar = 50um. (h) 470 Boxplots showing ratio of nuclear to cytoplasmic mCherry intensity in fat bodies. Lower ratio indicates 471 higher insulin activity. ( $n=10)$

472 Bar plots show mean and error bars indicate standard deviation. AEL= after egg-laying. $* *=p<0.01, n s=$ $473 \mathrm{p}>0.01$, (b-e) Student's t-test and $(\mathrm{f}, \mathrm{h})$ two-tailed Mann-Whitney $U$ test.

475 Figure 4. DILPs and AKH colocalize in corpora cardiaca dense-core vesicles.

476 (a) Schematic representation of experimental approach.

477 Electron micrograph of

478 (b) the CC soma (epon-embedded sections). $\mathrm{N}=$ nucleus, $\mathrm{G}=$ Golgi complex, ER = endoplasmic reticulum, 479 and $\mathrm{DCV}=$ dense-core vesicle. Scale bar $=500 \mathrm{~nm}$.

480 (c) Part of cytoplasm in the CC soma (methylacrylate sections). DILP5 labelled with immunogold particles of $4815 \mathrm{~nm}$ diameter. Dashed red line indicates area occupied by DCVs. Scale bar $=500 \mathrm{~nm}$ (d) Quantification of 482 gold particle density (no. of particles/um²) from DCV area (dashed red line) or rest of the cytoplasm (solid 483 black line) from multiple acquisitions $(n=4)$. Bar plot indicates mean. 
484 High-resolution electron micrographs of

485 (e) a multi-vesicular body (MVB, e.i) and a dense-core vesicle(DCV, e.ii) in the CC soma. DILP2 and DILP5 are 486 stained by $10 \mathrm{~nm}$ (closed arrow) and 5nm (open arrow) immunogold particles respectively. Scale $=100 \mathrm{~nm}$.

487 (f) DCV area (f.i) stained for DILP2 (10nm gold particle) and AKH (5nm gold particle). Dashed blue box 488 outlines a single DCV (f.ii) magnified on the right, DILP2 (closed yellow arrow) and AKH (open gray arrow).

489 Scale bar $=100 \mathrm{~nm}$.

490 (g) Histogram showing immunogold particle size vs. frequency distribution in (f), highlighting relative 491 abundance of DILP2 (yellow) and AKH (gray).

Figure 5. Corpora cardiaca DILPs enable starvation-induced accelerated pupariation.

495 (a) Experimental setup for larval starvation

496 (b-c) Means of percentage pupariation connected across timepoints for the indicated genotype- starved

497 (dashed line) or kept feeding (solid line) as shown in (a). Fed controls are the same as shown in Figure 3a.

$498 \mathrm{n}=6$ for each curve; $\mathrm{p}$-value from two-way ANOVA with repeated measures and Bonferroni's post $\mathrm{t}$-test for

499 multiple comparisons. Error bars indicate + /- S.D. ${ }^{* *}=p<0.01$ and $n s=p>0.01$

500 (d,f) CC soma of genotype (d) $a k h>w 1118$ and (f) $a k h>K I R 2.1$ stained for HRP(d.i,d.iv, f.i and fiv; gray) and

501 DILP2 (d.ii, d.v, f.ii and f.v; green). KIR2.1 expression inactivates neurons through hyperpolarization. Scale 502 bar $=10 u m$.

$503(\mathrm{e}, \mathrm{g})$ Average fluorescence intensity of DILP2 normalized to the mean of fed controls. Each point represents 504 a single CC soma. $n=3-5$.

505 (h-i) DILP2-HA-FLAG concentration in larval hemolymph of indicated genotype using driver line akh506 gal4,dilp2-HF (n=4-5).

507 (j) Fat body of indicated genotype stained for nuclei (j.i and j.ii; blue) and mCherry (j.ii and j.iv; red). White 508 dashed outlines show the position of some nuclei in the mCherry channel. Scale bar $=50 u m$. $(\mathbf{k})$ Boxplots 509 showing ratio of cytoplasmic to nuclear mCherry intensity in fat cells $(n=5)$.

510 (I) Prothoracic gland of indicated genotype stained for nuclei (I.i, I.iii, I.v and I.vii; blue) and mCherry (I.ii, I.iv, 511 I.vi and I.viii; red). White dashed outlines show the position of some nuclei in the mCherry channel. Scale 512 bar $=10 \mathrm{um}$. (k) Boxplots showing ratio of nuclear to cytoplasmic mCherry intensity in prothoracic gland 513 cells. $n=4-8$.

$514 \mathrm{AEL}=$ after egg-laying. ${ }^{* *}=p<0.01, \mathrm{~ns}=\mathrm{p}>0.01$, (e-g) Student's t-test and $(\mathbf{h}, \mathbf{i}, \mathbf{k}, \mathrm{m})$ two-tailed Mann-Whitney $515 \cup$ test. 


\section{Materials and methods}

\section{Flies}

525 All fly lines/crosses were maintained and grown on standard media containing cornmeal, molasses, agar

526 and yeast extract under a $12 \mathrm{hour}$ light/dark cycle at $25^{\circ} \mathrm{C}$ (unless explicitly stated otherwise).

527 List of fly strains used

\begin{tabular}{|c|c|c|}
\hline Strain & Source & Identifier \\
\hline w1118 & BDSC & \#5905 \\
\hline IPC-Gal4 (Dilp2-Gal4) & BDSC & \#37516 \\
\hline CC-Gal4 (akh-Gal4) & $\begin{array}{l}\text { (Tennessen, Baker, Lam, Evans, \& } \\
\text { Thummel, 2011) }\end{array}$ & NA \\
\hline dFOXO-mCherry & (Kakanj et al., 2016) & NA \\
\hline gDilp2-HA-FLAG & (Park et al., 2014) & NA \\
\hline UAS-SYT1-GFP & BDSC & \#6926 \\
\hline UAS-KIR2.1-GFP & BDSC & \#6596 \\
\hline UAS-Dcr2 & BDSC & \#24651 \\
\hline UAS-DILP2-RNAi & BDSC & \#31068 \\
\hline UAS-DILP5-RNAi & BDSC & \#31378 \\
\hline UAS-InR-RNAi & BDSC & \#31037 \\
\hline UAS-IMPL2RNAi & BDSC & \#64936 \\
\hline DILP-2,3,5,7 knock-out & BDSC & \#30889 \\
\hline ap-Gal4, tub-Gal80TS & Spannl et. al., 2020 & NA \\
\hline
\end{tabular}

528

529 Fly strains dilp2-Gal4,>dcr2, akh-Gal4,Foxo-mCherry, akh-Gal4, gdilp2-HF and ap-Gal4, tub-Gal80TS, Foxo-

$530 \quad$ mCherry were generated in this paper by crossing the above lines and are available upon request. 


\section{Developmental timing and starvation}

533 Fly crosses were fed fresh yeast paste and allowed to lay eggs for 3 hours on apple-juice agar plates.

534 Following egg-laying, the plates were stored at $25^{\circ} \mathrm{C}$. After $18-24$ hours of incubation, freshly hatched L1

535 larvae were collected in PBS in intervals of 2 hours. In order to prevent overcrowding, no more than 20-30

536 larvae were transferred into a single food vial using a micropipette and shifted to the incubator at $25^{\circ} \mathrm{C}$ with

537 12/12 day-night cycle. At specified time after egg-laying, larvae were carefully washed out of the food using

538 PBS. Subsequently they were quickly transferred to 1\% agar vials (same quantity as food). Fed controls were

539 not washed out, and left to feed in their original vials. Both starved and fed control vials were returned to

540 the same incubator.

541 For pupariation timing, number of pupae including white pre-pupae were manually counted at fixed

542 intervals as indicated. Counting was stopped when there was no increase for 3-4 consecutive time points.

543 The same dataset have been used in Figure 3a, and to depict fed controls in Figure 5b,c .

544 For all other starvation experiments, starved and fed control larvae were washed out of the agar/food with

545 PBS, rinsed and used in respective assays.

546

\section{Weight measurements}

548 Larval weight: At specified developmental stage, larvae were collected from food/agar in PBS, washed 3x

549 times to remove stuck food and cold-anesthetized. Female larvae were selected under the microscope to

550 prevent sex-dependent size variation. Cohorts of 13-15 larvae were blotted on a tissue paper (to ensure no

551 extra liquid) and transferred to a pre-weighed $1.5 \mathrm{ml}$ micro-centrifuge tube using forceps. The tube with

552 larvae was then weighed using a fine balance (Sartorius).

553 Adult weight: Virgin females (2 days after eclosion) were anesthetized and collected in pre-weighed $1.5 \mathrm{ml}$

554 micro-centrifuge in cohorts of 10 flies per tube. The tube with adult flies was weighed using a fine balance

555 (Sartorius).

556 Average weight in each case was calculated by dividing the weight difference with the number of larvae/flies

557 in each tube.

558

559 Size determination

560 Adult wing area: Females (1 day after eclosion) were collected and stored in isopropanol. Prior to dissection,

561 flies were transferred to a cavity dissection-glass. Care was taken to keep flies submerged in adequate 562 isopropanol at all times. One wing from each fly was dissected using fine forceps (Dumont, No.5-SA) and

563 transferred to a glass slide along with a small amount of isopropanol (in forceps due to capillary action). It

564 is important to keep wings flat on the slide surface. This can be achieved by repeatedly moistening the wings 
565 with isopropanol, using forceps. Once the desired number of wings were obtained, 30ul of Euparal (Roth,

566 cat\#7356.1) was pipetted on the slide. Next, a glass coverslip (22 mm/22mm/17um) was placed covering

567 the entire area on the slide occupied by wings. To ensure absence of air bubbles and minimum displacement

568 in the wings, it is important to place the coverslip starting from one edge. Following mounting, the slides

569 were left to dry for 2 hours prior to imaging. Single wing images were taken using Zeiss Sv11 dissection

570 scope fitted with a CCD camera (Jenoptik ProgRes C10).

571 Pupal volume: Pupae (24-30-hours APF) were collected using a wet brush. Following which, they were 572 washed with PBS to dislodge attached food and glue. Each pupa was then transferred to a single well of a 573 transparent 24-well plate and imaged using Zeiss Sv11 dissection scope fitted with a CCD camera (Jenoptik

574 ProgRes (10). Sex determination was done post-eclosion and only females were taken for analysis.

\section{Dilp2-HF ELISA}

Nunc MaxiSorp Flat-Bottom plates (Thermo Fisher Scientific) were coated with anti-FLAG M2 antibody

578 (Sigma, cat\#F1804) diluted in BupH Carbonate-Bicarbonate Buffer (Thermo Fisher Scientific, cat\#28382)

579 overnight at $4^{\circ} \mathrm{C}$. The plates were washed $2 x$ in wash buffer - PBS $+0.2 \%$ Tween20 (Sigma, cat\#P7949) and

580 blocked in $2 \%$ filter-sterilized BSA in PBS overnight at $4^{\circ} \mathrm{C}$. $1 \mathrm{~mL}$ of hemolymph was collected by bleeding 5 -

5818 heterozygous gDilp2-HF larvae on a clean cavity slide. The hemolymph was diluted in $55 \mathrm{~mL}$ PBS and

582 centrifuged at 10,000rpm for 10 minutes to remove hemocytes and debris. $50 \mathrm{~mL}$ supernatant and $1 \mathrm{~mL}$ of

583 anti-HA-HRP antibody (Roche, cat\#12013819001) solution (1:500) was incubated in the coated wells

584 overnight at $4^{\circ} \mathrm{C} .100 \mathrm{ml}$ of 1-Step-Ultra TMB-ELISA Substrate (Thermo Fisher, cat\#34028) was pipetted to

585 each well and incubated for 30 minutes in dark at room temperature. Reaction was stopped by adding 100

$586 \mathrm{ml}$ of $2 \mathrm{M}$ sulfuric acid per well. The samples colorized instantly and the absorbance was measured

587 immediately at 450nm using a plate reader (BMG Labtech). Standard curve was generated by self-generated

588 FLAG(GS)HA peptide. The detailed method is described in Park et al., (2014).

589

590 Immunofluorescence

591 Freshly dissected tissue in PBS was fixed with 4\% paraformaldehyde (cat\#16005, Aldrich) for 20 minutes.

592 After fixation, tissues were thoroughly washed 3-4 times with PBS. The following protocol was used 593 depending on tissue type.

594 Brain, ring gland and fat body: Tissues were permeabilized with PBS containing 0.2\%(v/v) Triton X-100(Serva

595 Electrophoresis $\mathrm{GmbH}$, cat\#37240.01) for 2 hours at room temperature with shaking. Primary incubation 596 with antibody was done overnight in incubation buffer- PBS with $0.2 \%$ Triton X-100 (PBST) and $1 \mathrm{mg} / \mathrm{ml}$ 597 bovine serum albumin (BSA) (Sigma, cat\#A2153). Incubation was followed by $3 \times 15$ minute washes with the 
598 same incubation buffer without antibodies. Secondary incubation was done with antibody diluted in 599 incubation buffer supplemented with $4 \%(\mathrm{v} / \mathrm{v})$ normal goat serum (NGS) overnight at $4^{\circ} \mathrm{C}$. Tissues were then

600 washed 4 X15 minutes in 0.2\%PBST and mounted on glass slides using VectaShield $\mathrm{H}-1000$ (cat\# H-1000-10)

601 mounting medium.

602 Wing discs: Discs were permeabilized with PBS containing 0.05\%(v/v) Triton X-100 supplemented with

$6031 \mathrm{mg} / \mathrm{ml}$ bovine serum albumin (BSA) and 250mM NaCl for 3X10 minutes at room temperature with shaking.

604 Primary incubation with antibody was done overnight in incubation buffer- PBS with 0.05\% Triton X-100

605 (PBST) and $1 \mathrm{mg} / \mathrm{ml}$ bovine serum albumin (BSA). Incubation was followed by $3 \times 15$ minute washes with the

606 same incubation buffer without antibodies. Secondary incubation was done with antibody diluted in

607 incubation buffer supplemented with 4\%(v/v) normal goat serum (NGS) for 2-3 hours. Wing discs were then

608 washed $3 \times 15$ minutes in 0.1\%PBST and mounted on glass slides using VectaShield $\mathrm{H}-1000$ mounting

609 medium.

610 All washing and incubation steps were performed on a shaker. Fat bodies from late larval stages disintegrate

611 easily and requires extra care while handling, preferably shaking at a low rpm.

612

613 List of primary antibodies

614

\begin{tabular}{|l|l|l|l|}
\hline Antibody & Host species & Dilution & Source \\
\hline Dilp2 & Rat & $1: 400$ & (Géminard et al., 2009) \\
\hline Dilp5 & Rat & $1: 1000$ & This paper \\
\hline Dilp5 & Rabbit & $1: 500$ & (Géminard et al., 2009) \\
\hline Akh & Rabbit & $1: 800$ & $\begin{array}{l}\text { (Isabel,Martin,Chidami, } \\
\text { Veenstra,\&Rosay, 2005) }\end{array}$ \\
\hline mCherry & Rabbit & $1: 500$ & Invitrogen \#PA5-34974 \\
\hline GFP & Rabbit & $1: 500$ & Invitrogen \#A111022 \\
\hline IMPL2 & Rabbit & $1: 1000$ & (Bader et al., 2013) \\
\hline
\end{tabular}

615 Secondary antibodies/dyes used were DAPI (1:2000 from 5mg/ml stock, Roche), DyLight 405 goat anti-HRP

616 (1:500, Jackson IR, cat\#123-475-021), AlexaFlour-488 goat anti-rabbit (1:1000, Invitrogen \#A11034),

617 AlexaFlour-568 goat anti-rabbit (1:500, Invitrogen \#A11036) and AlexaFlour-647 goat anti-rat (1:500,

618 Invitrogen \#A21247).

619

620 Confocal Light microscopy 
621 Three imaging setups have been used for fixed samples-

622 1. Olympus FV1000 laser scanning confocal microscope on Olympus BX61 inverted stand and driven by

623 FV10-ASW 1.7 software. Brain and ring gland whole mounts were imaged with Olympus UApochromat 40x

624 1.3NA oil immersion objective. Corpora cardiaca cells were imaged with Olympus UApochromat 60x 1.35

625 NA oil immersion objective.

626 2. Andor spinning disc confocal microscope on Olympus IX81 inverted stand using Andor iXON 897 EMCCD

627 camera with Yokogawa CSUX1 scanhead, running on Andor software. Some fat bodies were imaged with

628 Olympus U Plan SApo 60x/ 1.35NA oil immersion objective.

629 3. Olympus FV3000 laser scanning confocal microscope on Olympus IX83 inverted stand and driven by

630 FV31S-SW acquisition software. Corpora cardiaca cells and wing discs were imaged with Olympus

631 UPLXApochromat 60x 1.42 NA oil immersion objective.

632

633 Electron microscopy

634 Morphology: Dissected brain-ring gland complexes were initially fixed in PBS with 2\%PFA (cat\#16005,

635 Aldrich) and 1\% glutaraldehyde (cat\#G5882, Sigma) for 2 hours at room temperature followed by overnight

636 incubation at $4^{\circ} \mathrm{C}$. Then the samples were post-fixed with 1\% OsO4 (cat\# 19190, EMS) and 1.5\% potassium

637 ferrocyanide (cat\#: P9387, Sigma-Aldrich) in water on ice for 1 hour. En bloc staining was done in the dark

638 with 1\% UA (cat\# 21447, Polyscience Europe $\mathrm{GmbH}$ ) in water at $4^{\circ} \mathrm{C}$ overnight. Following infiltration and

639 embedding, 70nm sections were cut on a Leica Ultracut UCT (Leica Microscopy systems) and picked up with

640 formvar (cat\# 15800, EMS) coated slot grids (cat\# G2010-Cu, EMS). All samples were then post-stained with

$6411 \%$ UA (cat\# 21447, Polysciences Europe GmbH) in water for 10 minutes and 0.04\% lead citrate (cat\# 17800,

642 EMS) for 5 minutes. Electron micrographs were obtained at 80kV using Morgagni (Emsis Morada CCD

643 camera, formerly Sys/Olympus) and 100kV using Tecnai 12 (TVIPS F416 camera) electron microscopes (both

644 from Thermo Fisher Scientific (formerly FEl/Philips)).

645

646 Immunogold labelling: Dissected brain-ring gland complexes were initially fixed in PBS with 4\% PFA and

$6470.05 \%$ glutaraldehyde for 20 minutes at room temperature. Post-fixation was done in 1:1 dilution of the 648 initial fixative overnight at $4^{\circ} \mathrm{C}$. Ring glands were then embedded with $12 \%$ gelatin (cat\# G-2500, Sigma649 Aldrich) in PBS and infiltrated with 2.3M sucrose (cat\# 21600, EMS) in PBS overnight at $4^{\circ} \mathrm{C}$. The sucrose 650 infiltrated Ring gland in gelatin was mounted on an aluminum pin and snap frozen in LN2 and cryosectioned 651 on a Leica Ultracut EM UC6 ultramicrotome (Leica Microscopy systems). 70nm Sections were picked up 652 using a $1: 1$ mixture of 2.3M sucrose and 2\% methyl cellulose (cat\# M-6385, Sigma-Aldrich) as a pick-up 653 solution and sections were transferred to carbon and formvar coated mesh grids (cat\# H100-Cu, EMS). After 
654 removal of gelatin by incubation on PBS at $37^{\circ} \mathrm{C}$, sections were labelled with rabbit-anti-Dilp2/Dilp5 or goat-

655 anti-AKH Antibody (diluted 1 to 20 or 1 to 100 from original antibody solution) followed by secondary

656 antibody coupled to 10-nm gold (British Biocell or Bbisolutions) and secondary fluorescence antibodies

657 (donkey-anti-rat Alexa594 cat\# A-21209, Molecular Probes Europe B.V., goat-anti-rabbit Alexa488 cat\# A-

658 21206, Mol. Probes Europe B.V.) The sections were first imaged with epifluorescence on a Zeiss upright

659 ApoTome microscope with Axiocam506 camera to locate regions of interest in individual sections and

660 contrasted with a mixture of $1.9 \%$ methyl cellulose/0.3\% UA for $10 \mathrm{~min}$ on ice and the corresponding regions

661 of interest were observed by using a Morgagni or Tecnai12 electron microscope.

662

663 Image analysis and quantification

664 Whole cell: Individual cells from multiple samples were manually segmented and mean intensity was

665 measured using Fiji/ImageJ (Schindelin et al., 2012).

666 Co-localization : Single confocal images from two different channels were used in Coloc 2 plugin in

667 Fiji/ImageJ (Schindelin et al.,2012).

668 Nuclear and cytoplasmic intensity ratio: Regions of interested were manually drawn on respective tissues.

669 Nuclear to cytoplasmic intensity ratio was then calculated using a Fiji/ImageJ macro developed by Volker

670 Baecker at Montpellier RIO Imaging (Intensity Ratio Nuclei Cytoplasm Tool, RRID:SCR_018673).

671 Documentation can be accessed here. Same analysis parameters were used across samples compared.

672 Electron micrographs: For morphological sections, composite images were assembled in Fiji (Schindelin et

673 al., 2012) using ImageJ TrackEM2 plugin (Cardona et al., 2012). For immunogold labelling, regions of interest

674 were visually determined and gold particles were hand-counted in Fiji/ImageJ.

675 Wing area: Each wing blade was hand segmented and area measured in pixels using a self-written macro 676 on Fiji/ImageJ.

677 Pupal volume: Using a self-written Fiji/Imagej macro, an ellipse was fitted to the pupa. Volume was 678 calculated assuming an ellipsoid using the formula below (minor axis length as the height) -

679

$$
\text { Volume }=0.75 \pi *(\text { major-axis }) *(\text { minor-axis })^{2} \text {. }
$$

680

681 Data representation and statistical analysis

682 Data wrangling was done using Microsoft Excel and R-Studio (version 1.3.1093). R-Studio was also used for 683 plotting. Packages used in R- plyr, dplyr, ggplot2 and xlsx. Plots generated through R were then formatted

684 using Adobe Illustrator 2021. Illustrations were generated using BioRender (www.biorender.com).

685 Statistical analysis was done using Microsoft Excel, GraphPad Prism 5.0 and online through 686 https://www.socscistatistics.com. Information about sample sizes and statistical tests used in this study can 
bioRxiv preprint doi: https://doi.org/10.1101/2021.07.05.451134; this version posted July 6, 2021. The copyright holder for this preprint (which was not certified by peer review) is the author/funder, who has granted bioRxiv a license to display the preprint in perpetuity. It is made available under aCC-BY-NC-ND 4.0 International license.

687 be found in the respective figure legends. Detailed information on sample size and replicates have also been 688 summarized in Table 1 of the supplementary material.

689 
a
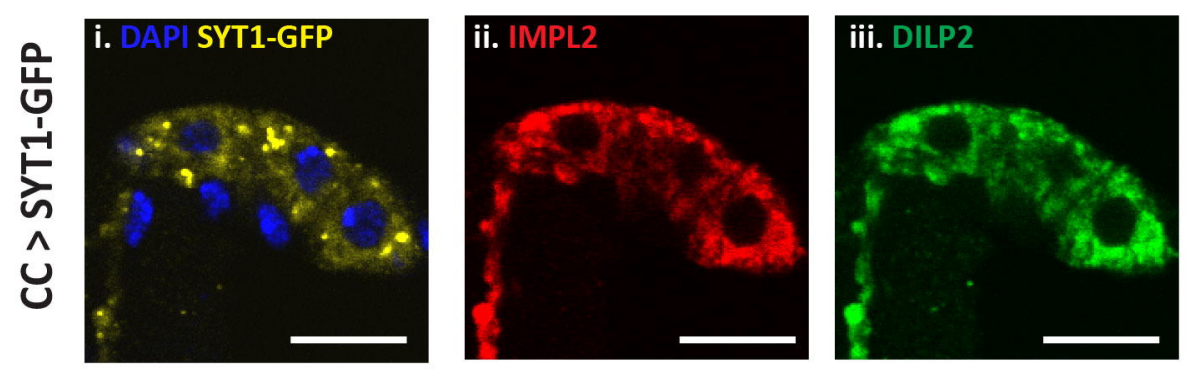

b

芯
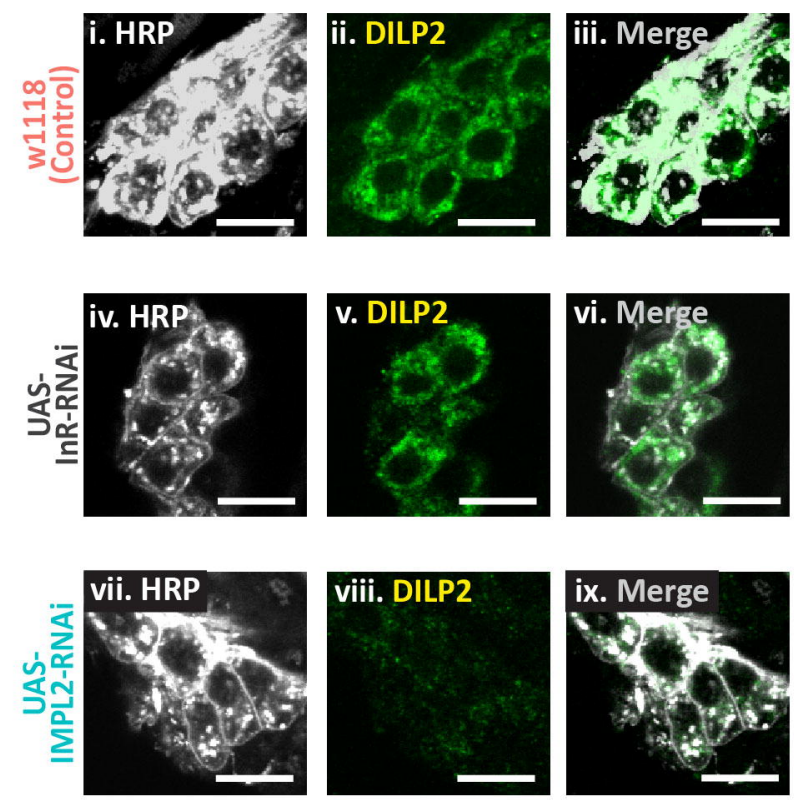

C

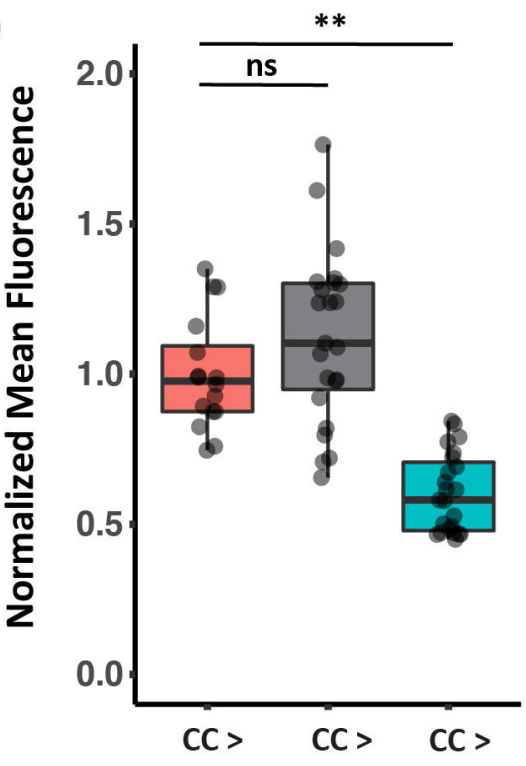

w1118 InR IMPL2

(Control) -RNAi -RNAi

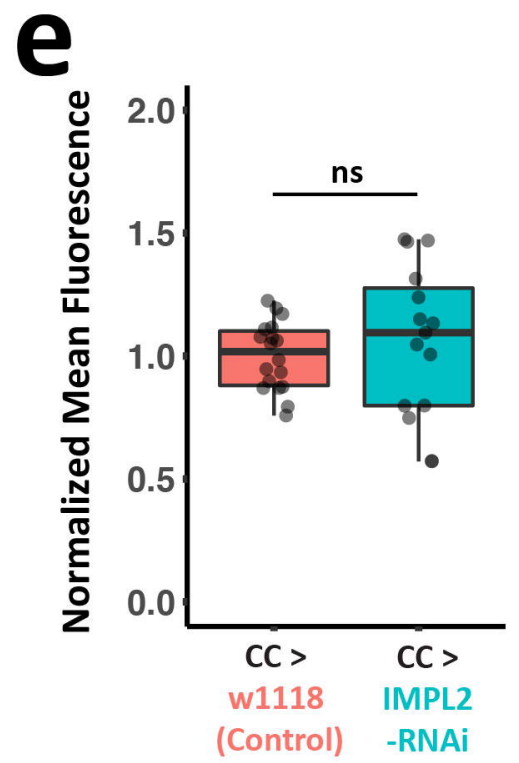


a

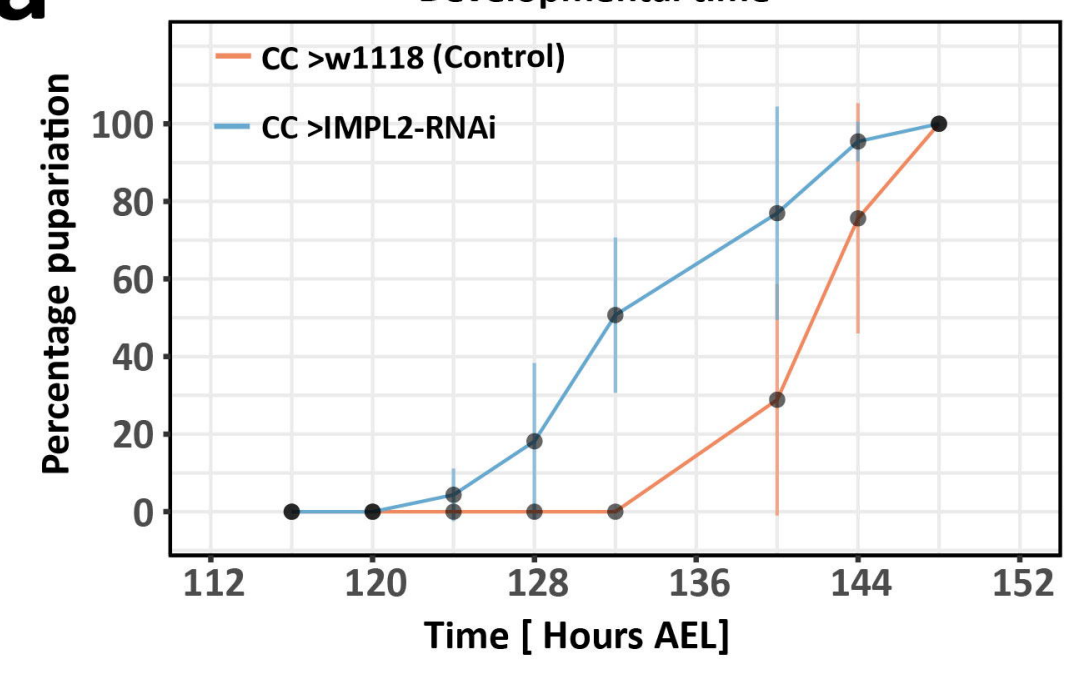

e

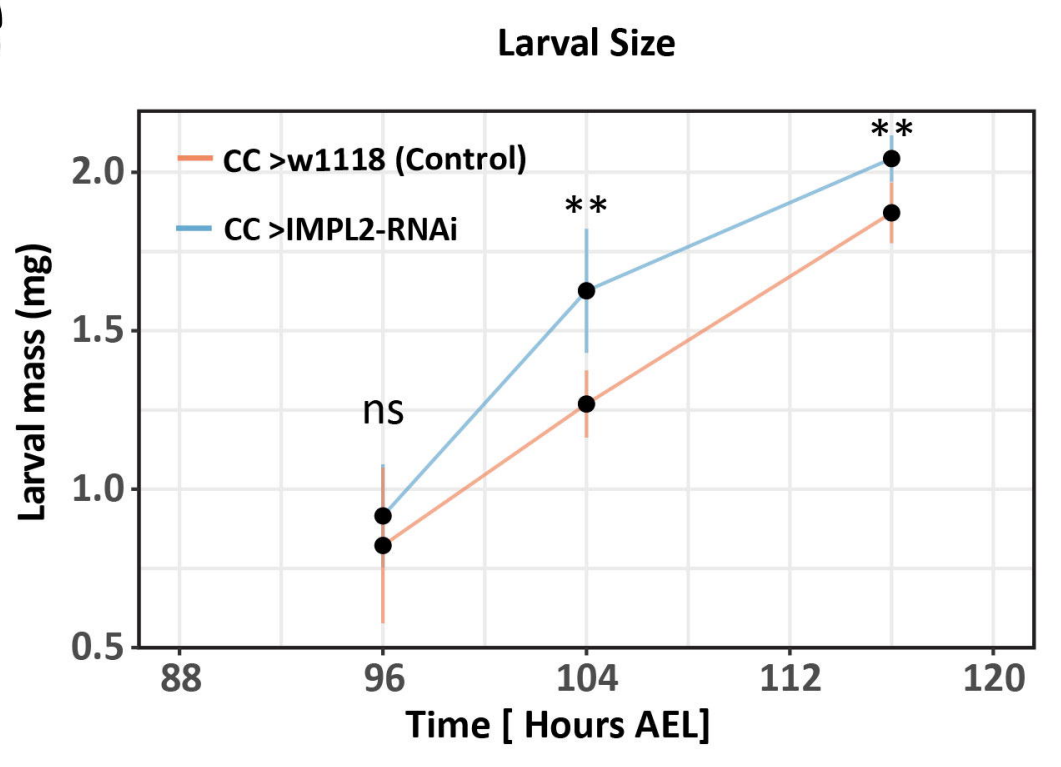

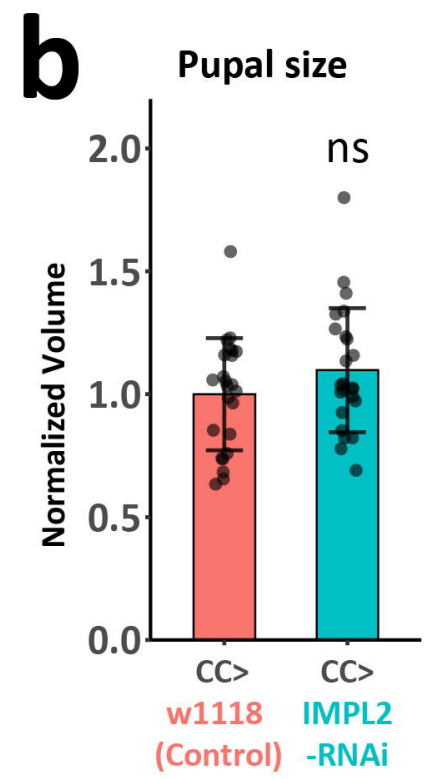

Circulating insulin

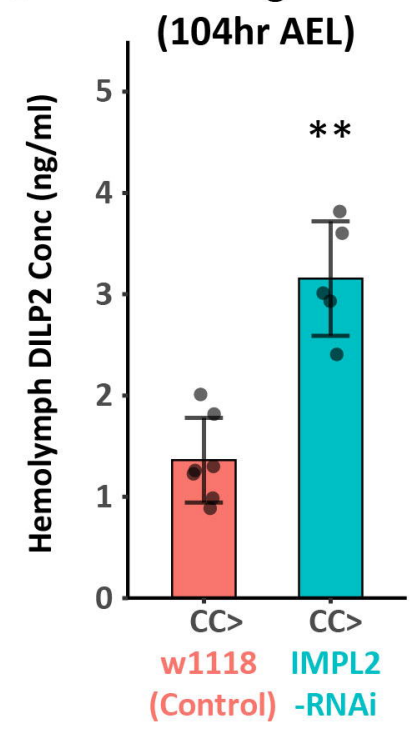

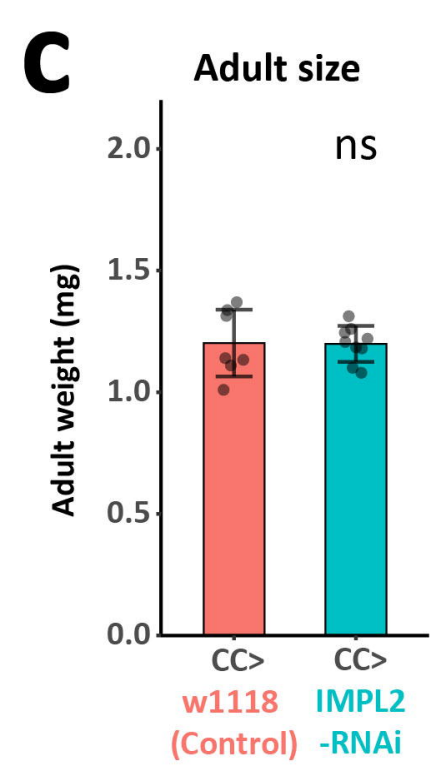

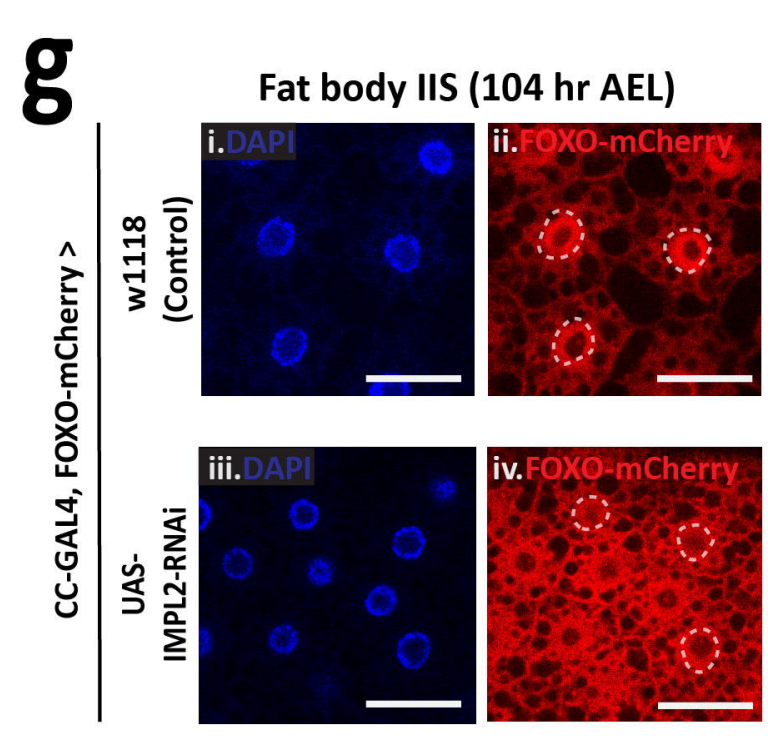

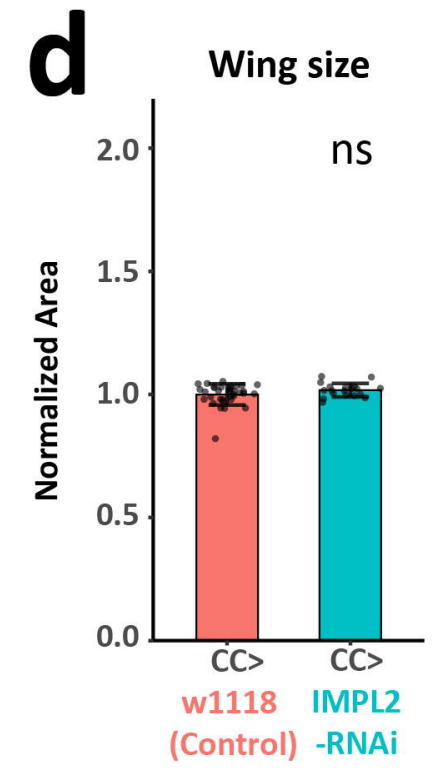

h

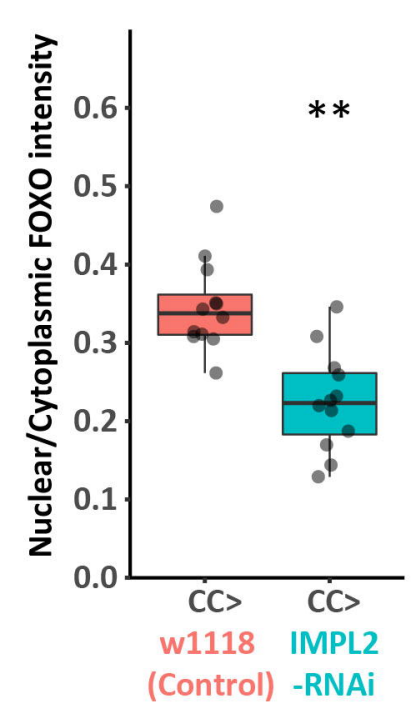




\section{a}

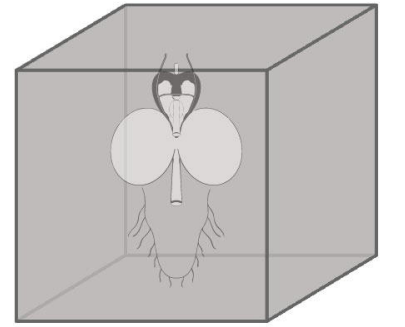

Tissue embedded in Epon(for morphology) or methylacrylate

(for immunogold staining)
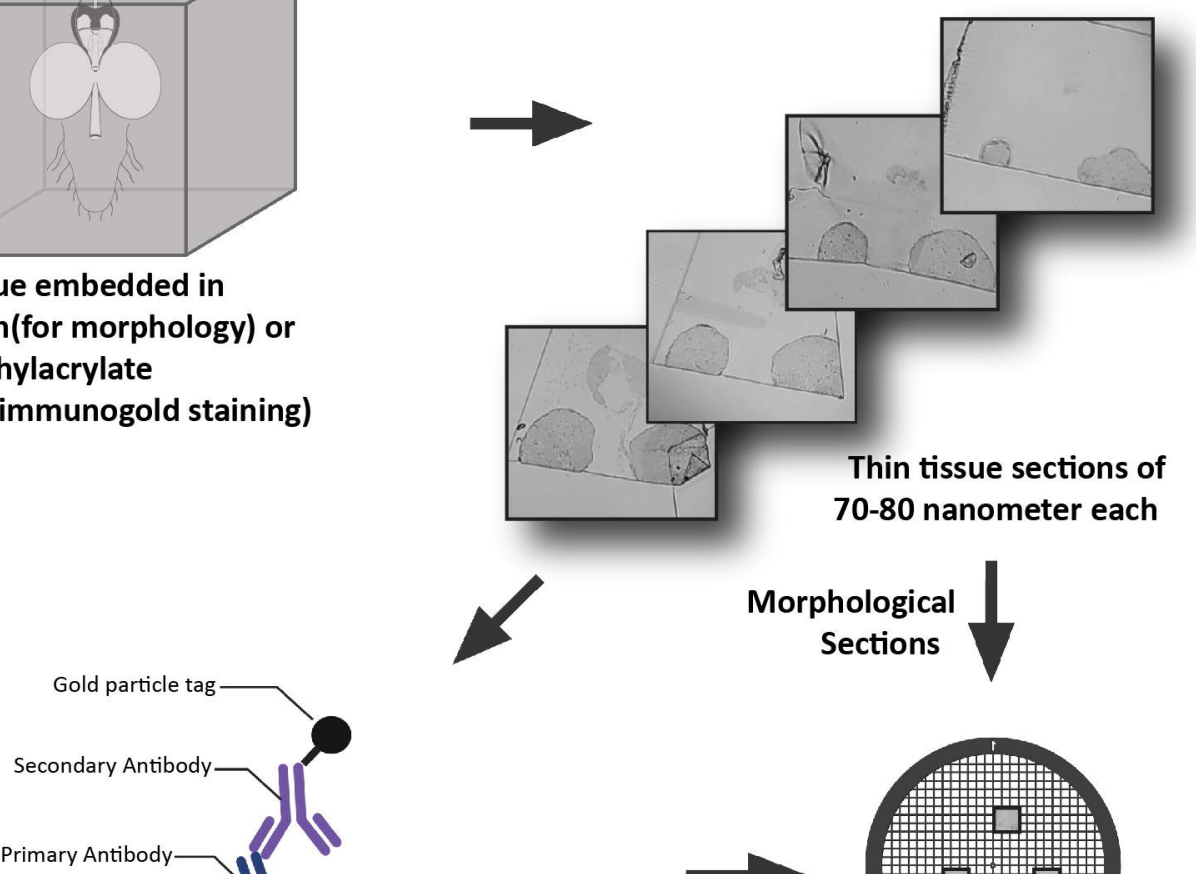

was not centifitigd by peef review) is the aistherblftutider, who has granted bio
available under aCC-BY-NC-ND 4.0

Immuno-gold staining

Transmission Electron Microscopy

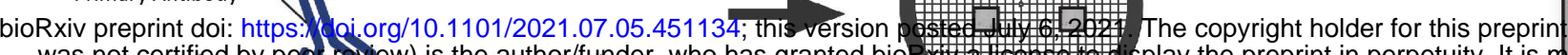

b

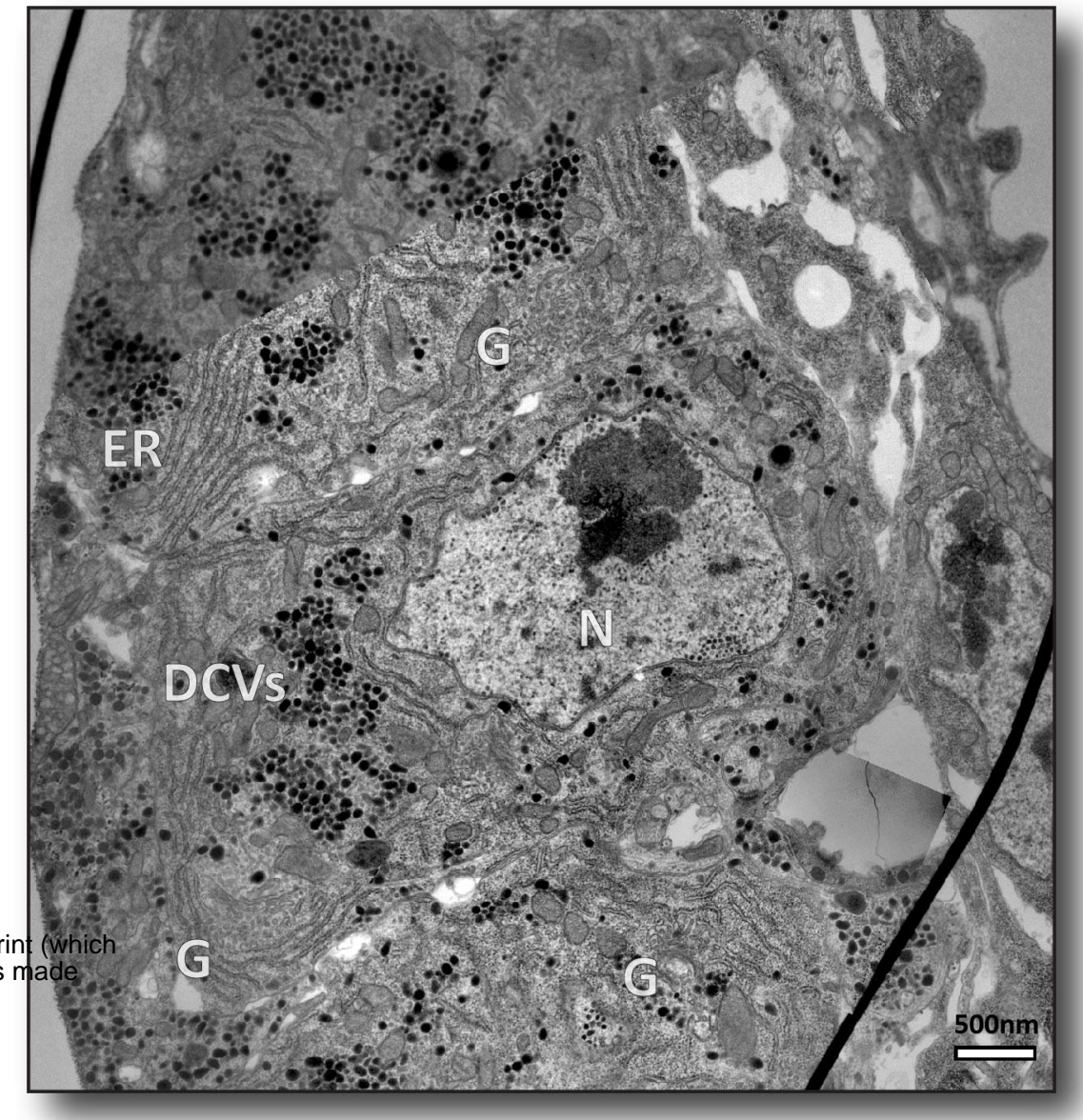

d
DILP5 - 5nm particles

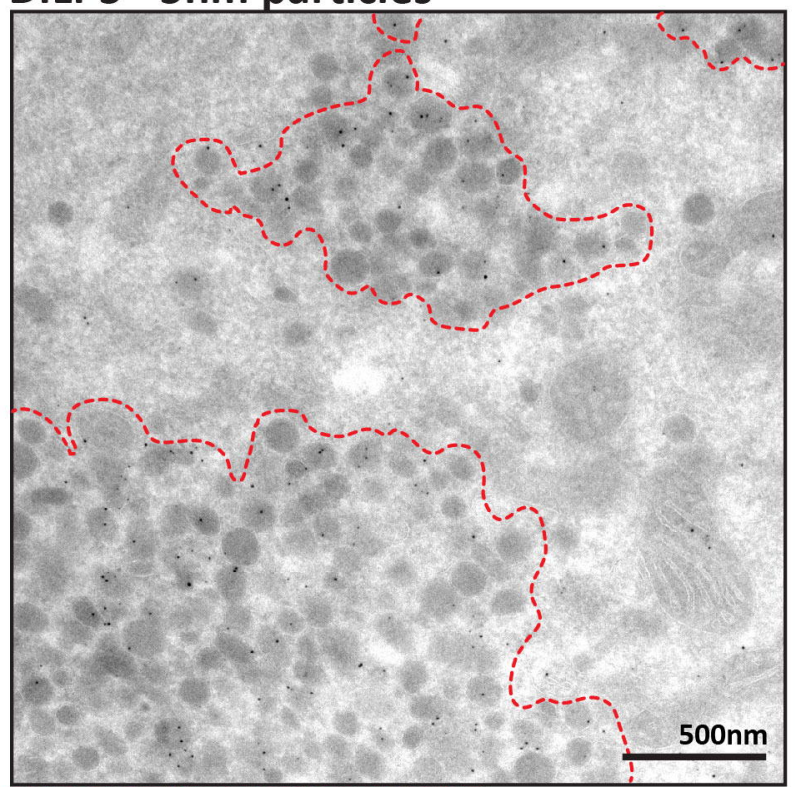

Dense-core vesicle area

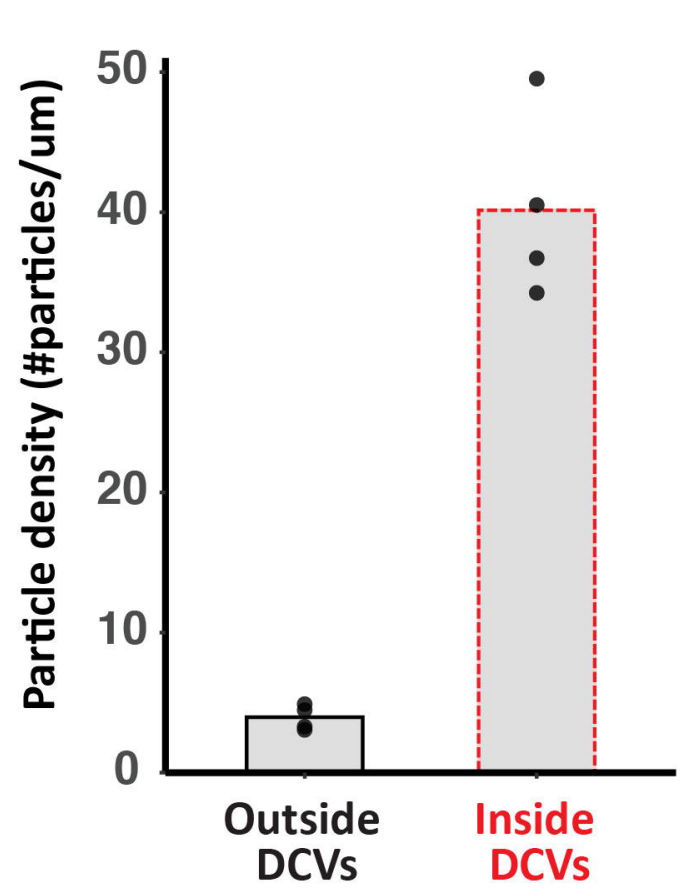

e
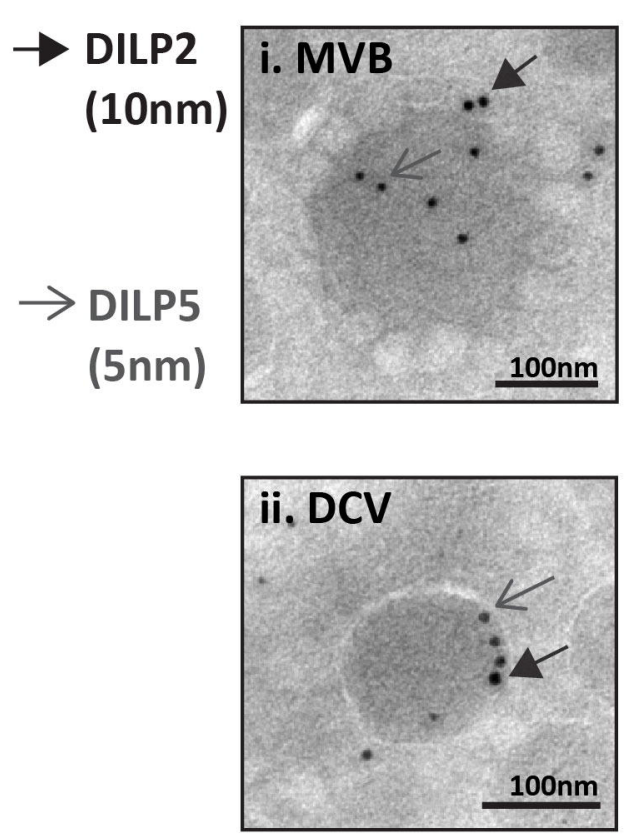

g

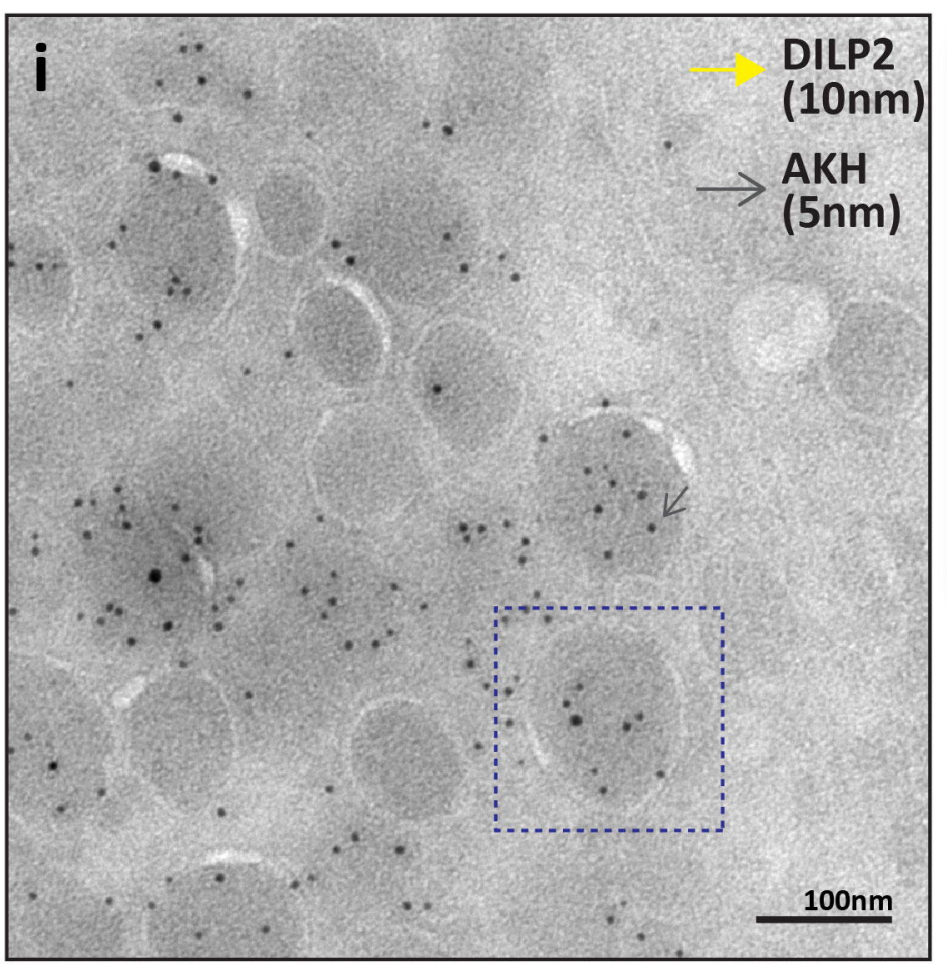

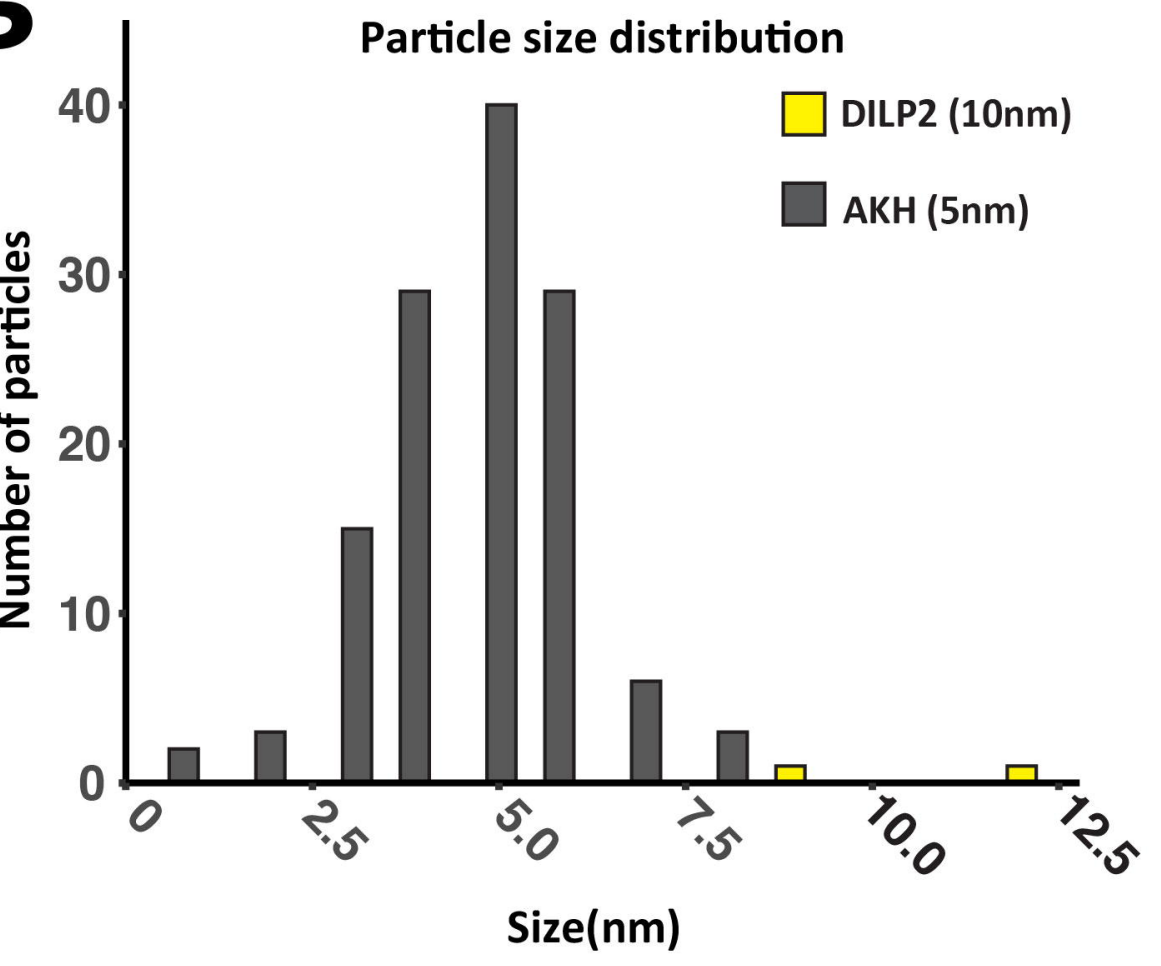



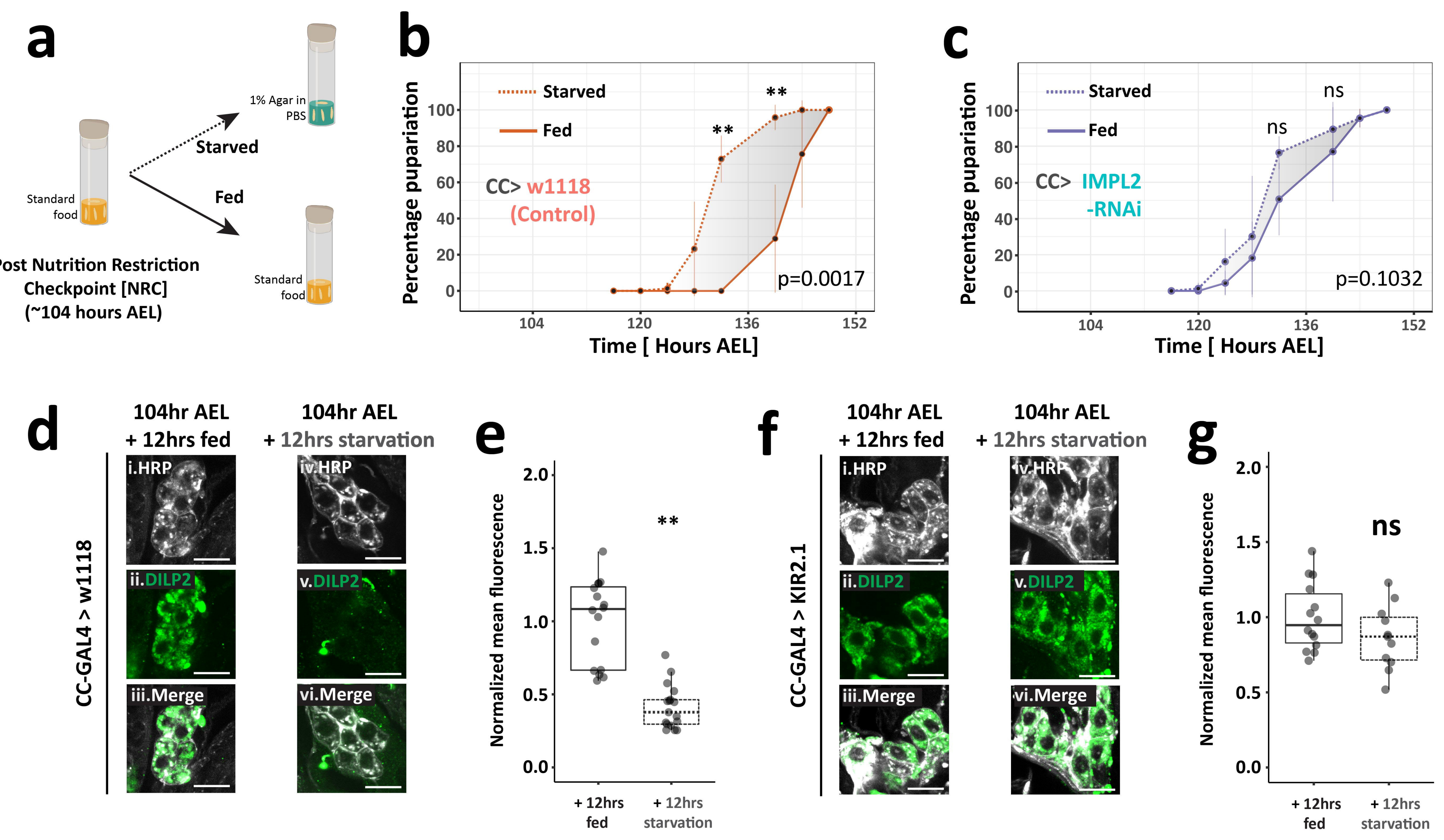

h

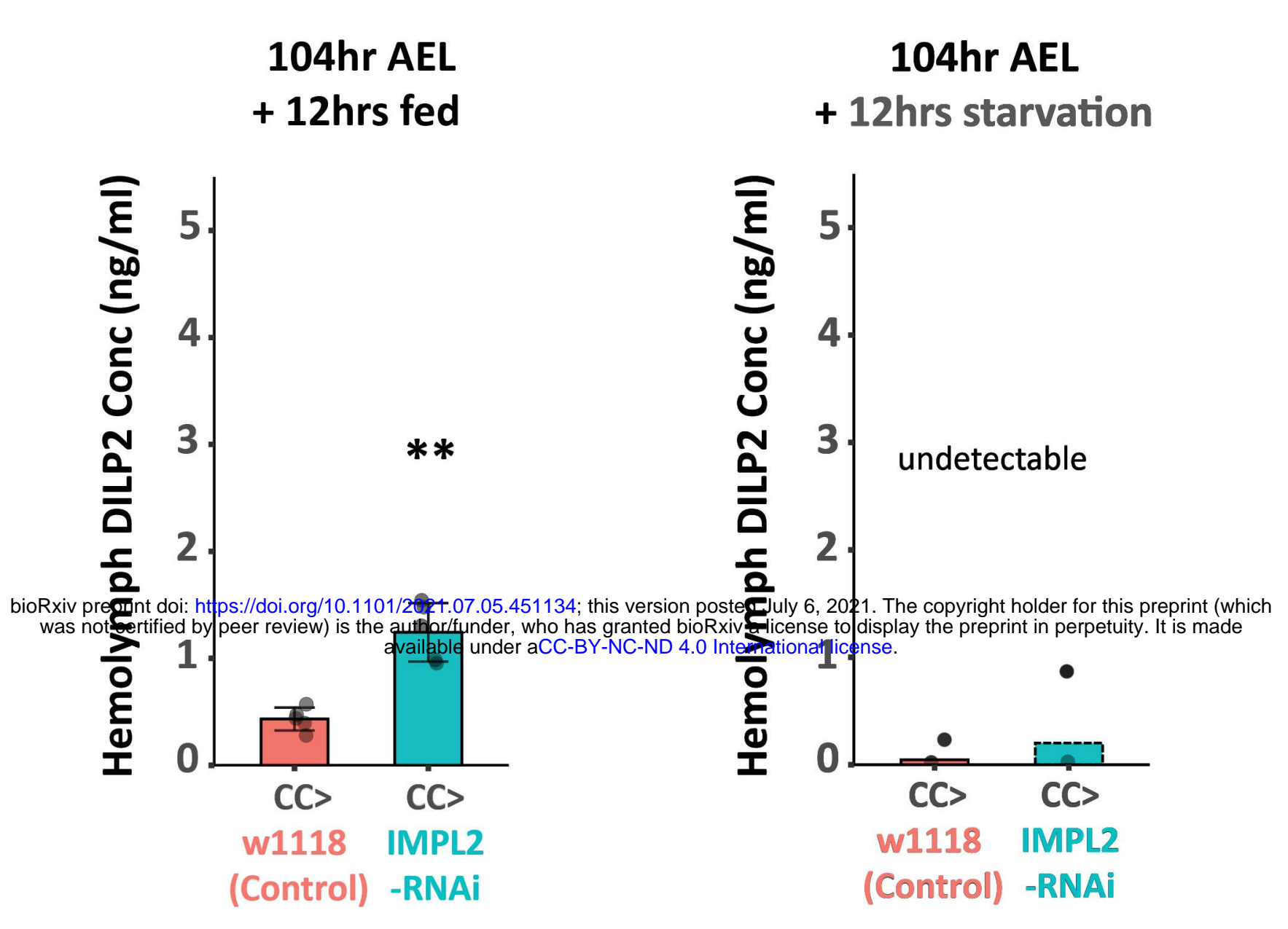

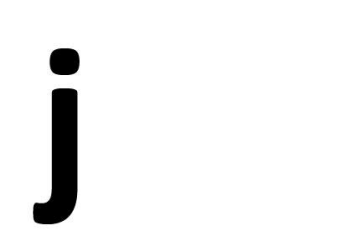

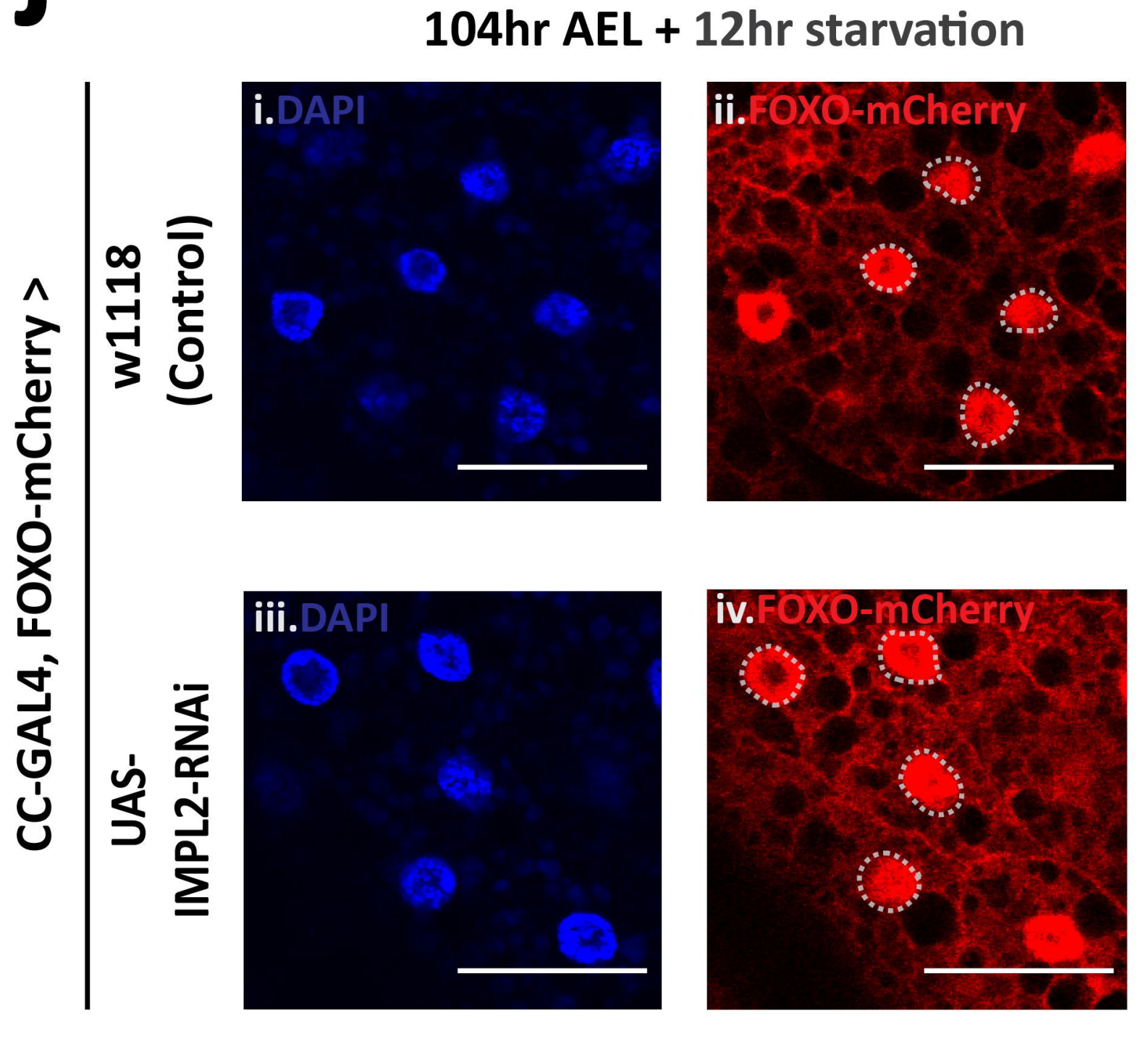

Fat body IIS

04hr AEL + $12 \mathrm{hr}$ starvation

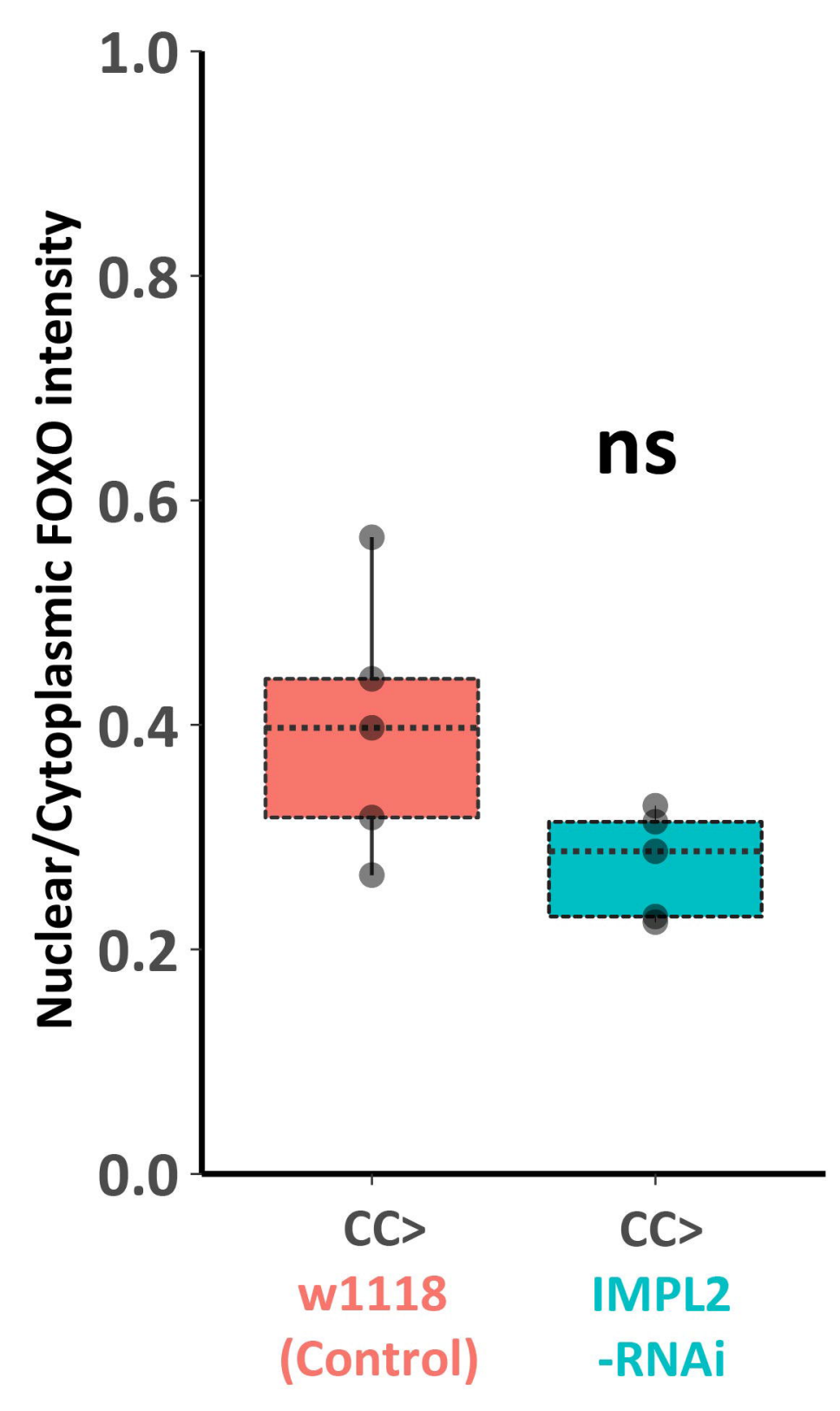

PG IIS activity

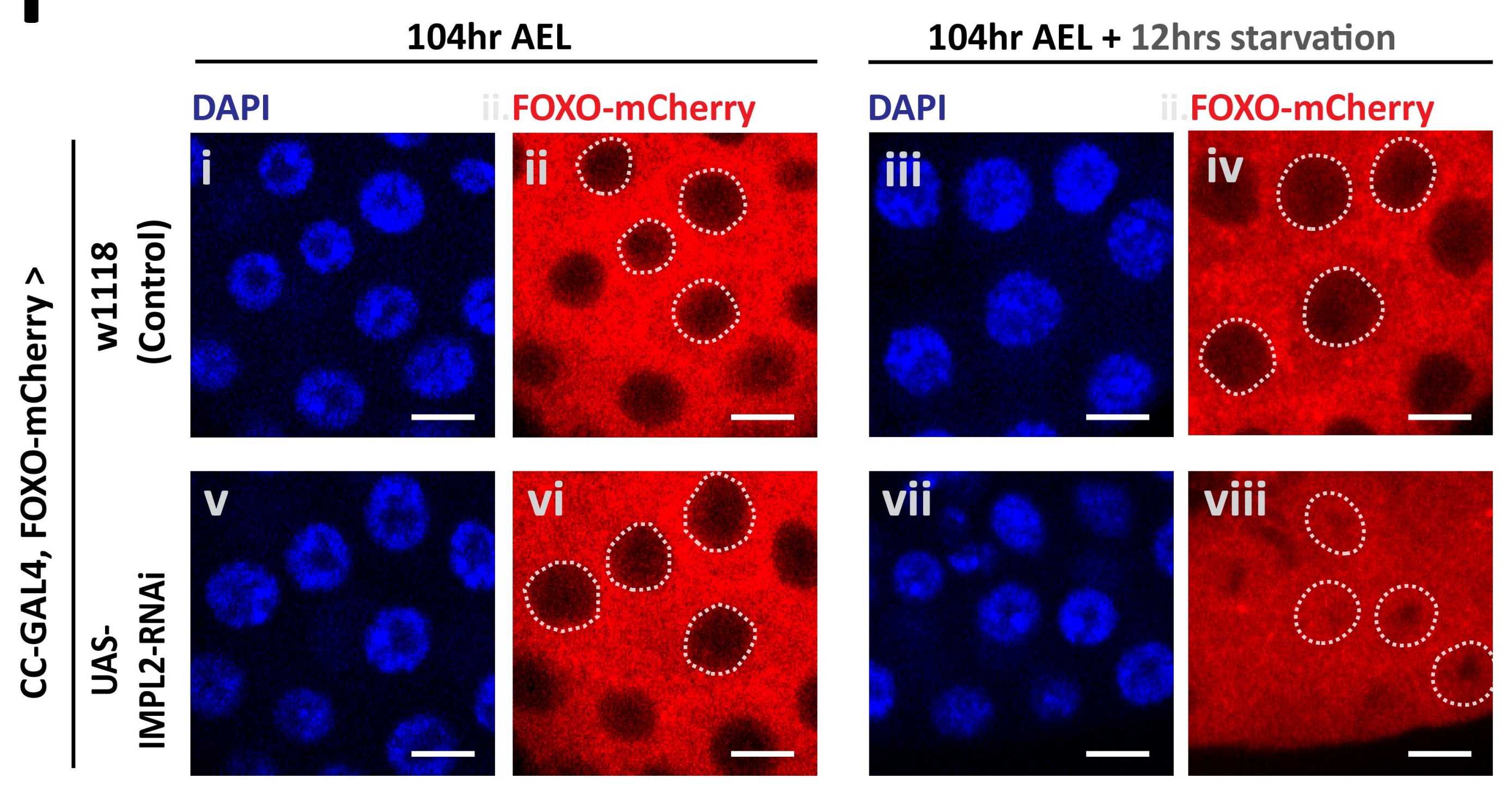

m

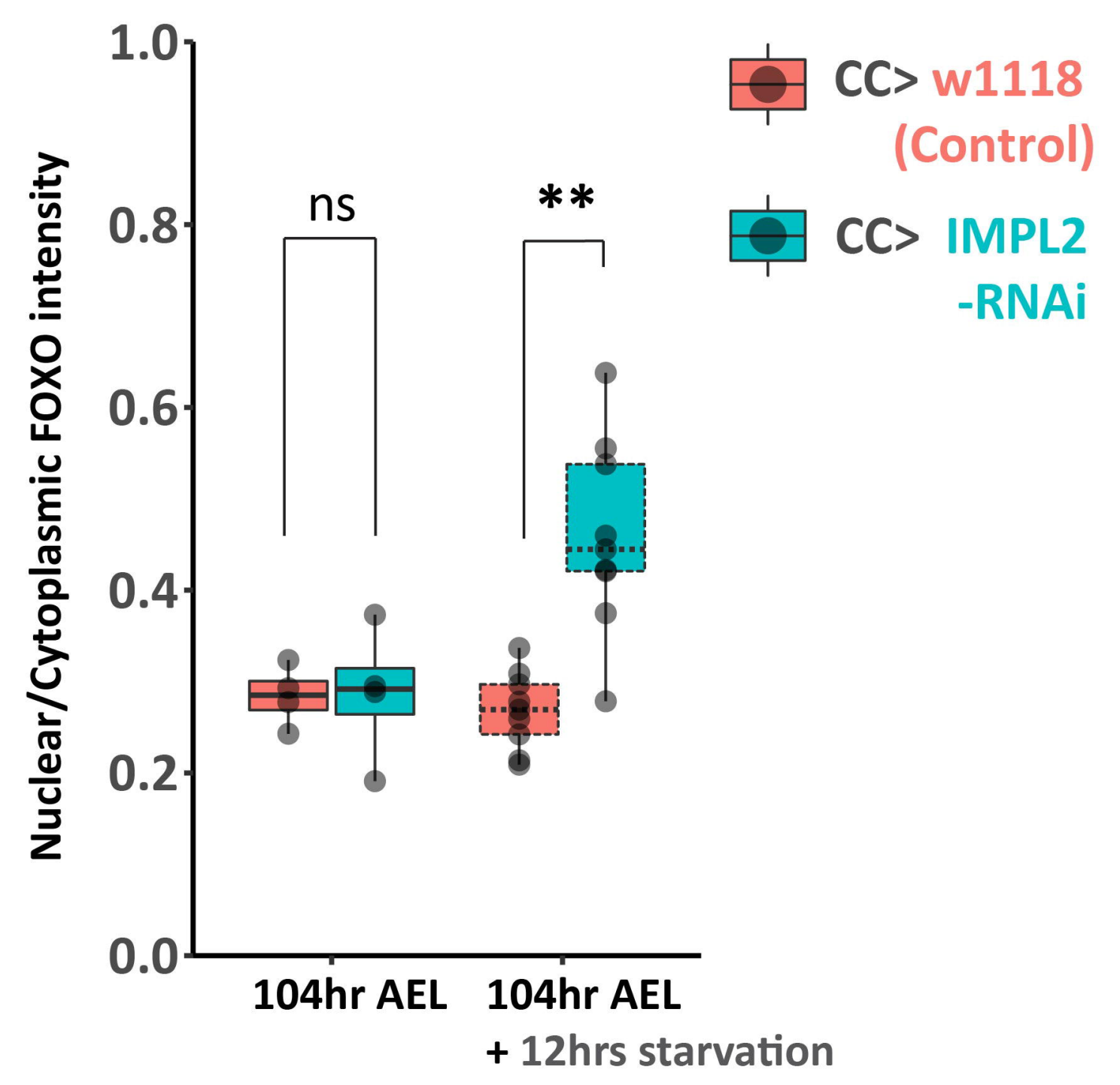

